\title{
OBERSTE KOMMANDOBEHÖRDEN UND DIENSTSTELLEN DER WEHRMACHT
}

Die schriftliche Überlieferung der Kommandobehörden, Stäbe, Truppenteile und Dienststellen der Wehrmacht aus der Zeit der Aufrüstung ab 1933 und dem Zweiten Weltkrieg ist zu einem großen Teil, insbesondere auch durch die Vernichtung der Bestände des Heeresarchivs in Potsdam durch einen Luftangriff im April 1945, nur sehr unvollständig. Sie wird bis auf kleinere Bestände im früheren Militärarchiv der DDR in Potsdam und Bestände des Kriegsarchivs der Waffen-SS in der Tschechoslowakei überwiegend im Bundesarchiv-Militärarchiv in Freiburg verwahrt und ist in der Übersicht DAS BUNDESARCHIV UND SEINE BESTÄNDE, 3. Aufl. 1977, S. 158-187, 203-263, 271-295, 299-340 hinreichend beschrieben. Im folgenden werden lediglich Bestände durch ergänzende Angaben oder Verweise berücksichtigt, die Quellen zur allgemeinen politischen Geschichte, vor allem zum Verhältnis zur NSDAP und zur zivilen Verwaltung, oder zur Geschichte einzelner Regionen im späteren Gebiet der Bundesrepublik Deutschland enthalten. Schriftgut der Wehrmachtgerichtsbarkeit ist im Abschnitt 3.2.2.2 behandelt. Ergänzungsüberlieferung findet sich häufig in Akten des Sachgebiets Zivile Reichsverteidigung der Behörden der allgemeinen inneren Verwaltung (Kapitel 2), die Gegenüberlieferung ziviler Rüstungsdienststellen wird im Abschnitt 6.1.4 nachgewiesen.

LiL: SCHRIFTEN ZUM STAATSAUFBAU 25, 26 und 31/32. 1939. - R. ABSOLON: Die Wehrmacht im Drituen Reich. 6 Bde. 1969 ff. - F. Frh v. SIEGLER: Die höheren Dienststellen der deutschen Wehrmacht 1933-1945. 1953. - B. MUELLER-HLLLEBRAND: Das Heer 1933-1945. 3 Bde. 1954-1969. - W. LOHMANN und H. H. HILDEBRAND: Die deutsche Kriegsmarine 1939-1945. 1956 ff. - G. TESSIN: Vertände und Truppen der deutschen Wehrmacht und Waffen-SS im Zweiten Weltkrieg 1939-1945. 17 Bde. 1967-1988.

\subsection{Oberste Kommandobehörden mit nachgeordneten zentralen Dienststellen}

\subsubsection{Adjutantur der Wehrmacht beim Führer BA-MA, Best. RW 8}

Der Bestand enthält nur Aktensplitter (15 Bde, 1935-1945), darunter Unterlagen zur Besetzung der Tschechoslowakei (,Fall Grün“, 2 Bde, 1938-1939), ein Brieftagebuch über Kurierpost (2 Bde, 1942-1945), die zwischen Hitler und Kaiser Wilhelm II. 1940 gewechselten Telegramme, Akten über die Betreuung der Angehörigen gefallener Ritterkreuzträger (3 Bde, 1943-1944) und privatdienstlichen Schriftwechsel des Heeresadjutanten Major Gerhard Engel (4 Bde, 1941-1943, Briefe von 1940 im Nachlaß N 118), Ergänzungsüberlieferung, u. a. zu seiner Aufzeichnung über Hitlers Ansprache vom 7. November 1937 über die Vorbereitung des Krieges, im NachlaB des Wehrmachtadjutanten (1934-1938) Friedrich Hoßbach (N 24).

Lit.: GUIDES to German Records. vol. 18. - F. HOSSBACH: Zwischen Wehrmacht und Hitler 1934-1938. 1965. - B. F. SMITH: Die Überlieferung der Hossbach-Niederschrift im Lichte neuerer Quellen. 1990. - H. v. KOTZE: Heeresadjutant bei Hitler 1938-1943. 1974. 


\subsubsection{Kommandant des Führerhauptquartiers BA-MA, Best. RW 47}

Überliefert sind lediglich das Kriegstagebuch für die Zeit vom 23. August 1939 bis 15. Juli 1942, das u. a. Begegnungen mit Mussolini und anderen ausländischen Politikern verzeichnet (6 Bde). stenographische Aufzeichnungen über 52 Lagebesprechungen zwischen Dezember 1942 und März 1945 und wenige Akten über Organisation und Unterbringung (7 Bde, 1942-1945). Die Niederschriften über Hitlers Tischgespräche im Führerhauptquartier, die im Auftrag des Leiters der Parteikanzlei von dessen Adjutanten Ministerialrat Heim und von Regierungsrat Dr. Picker angefertigt wurden, befinden sich in Privatbesitz (authentische Abschrift des Manuskripts von Picker im BA, Kl. Erw. 441).

Lit: LAGEBESPRECHUNGEN im Führerhauptquartier. Hrsg. von H. Heiber. 1962. - H. PICKER: Hitlers Tischgespräche im Führerhauptquartier 1941/42. 1965. - MONOLOGE im Führerhauptquartier 1941-1944. Hrsg. von Werner Jochmann. 1980.

\subsubsection{Oberkommando der Wehrmacht (OKW)}

BA-MA, Best. RW 2 bis RW 7, RW 19

Überlieferung des Reichswehr- (bis 1935) bzw. Reichskriegsministeriums, aus dessen Wehrmachtamt bei der Übernahme des Oberbefehls über die Wehrmacht durch Hitler 1938 das OKW hervorging und dessen übrige Funktionen auf die Oberkommandos von Heer und Kriegsmarine aufgeteilt wurden, liegt hauptsächlich als Vorakten in deren Schriftgut vor, daneben sind noch wenige Vorgänge aus dem Ministerbüro (Best. RW 1, u. a. Korrespondenz v. Blombergs und von Oberst bzw. Generalmajor v. Reichenau, Protokolle des Reichsverteidigungsrates) erhalten geblieben. Aus den zahlreichen Ämtern und Abteilungen des OKW konnten dagegen größere Teilbestände gebildet werden; sie werden ergänzt durch Dokumente aus dem Nürnberger IMT-Verfahren gegen die Chefs von OKW und Wehrmachtführungsstab, Keitel und Jodl (Ergänzungen dazu in den Nachlässen, Bestände N 54 und N 69, zu Jodl auch IfZ, Best. ED 115) und den Generalstab als verbrecherische Organisation und dem sog. OKW-Prozeß vor dem US-Militärgericht, in dem u. a. die Generale Warlimont und Reinecke, Chefs der Abteilung Landesverteidigung bzw. des Allgemeinen Wehrmachtamtes, und der Chef der Wehrmachtrechtsabteilung, Generaloberstabsrichter Lehmann, angeklagt waren.

Lit: GUIDES to German Records. vol. 7, 8, 17, 18. - KRIEGSTAGEBUCH des Oberkommandos der Wehrmacht (Wehrmachtfuhrungsstab) 1940-1945. 4 Bde. 1961-1965 (1982). - TRIALS of War Criminals. vol. $X$ und XI. 1950-1951. - W. HUBATSCH: Hitlers Weisungen für die Kriegführung. 1962. - K. MEHNER (Hrsg.): Die geheimen Tagesberichte der deutschen Wehrmachtführung im Zweiten Weltkrieg. 1984 ff. $-W$. GÖRLITZ: Generalfeldmarschall Keitel. 1961. - G. MUELLER: The forgotten Field Marshall Wilhelm Keitel. 1979. - W. WARLIMONT: Im Hauptquartier der deutschen Wehrmacht 1939-1945. 1962. - L. JODL: Jenseits des Endes. 1987.

Wegen ihrer Bedeutung für die allgemeine politische Geschichte sind davon folgende Teilprovenienzen des OKW zu berücksichtigen:

Wehrmachtrechtsabteilung in Best. RW 2 Den Hauptteil des Bestandes bilden die Unterlagen der Wehrmacht-Untersuchungsstelle für Verletzungen des Völkerrechts durch die Kriegsgegner im allgemeinen (20 Bde, 1940-1945), durch Polen (10 Bde, 1939-1940), die Westmächte (88 Bde, 19401945 , davon 25 betr. Vorgänge in einzelnen amerikanischen Kriegsgefangenenlagern, 13 betr. Vorfälle auf Kreta 1941-1942) und die Sowjetunion (99 Bde, 1941-1945). 
AuBerdem liegen Vorgänge über die Anwendung des Wehrrechts (10 Bde, 1936-1944) und über Rechtsfolgen der Kapitulation (4 Bde, 1945) vor.

Lit.: A. de ZAYAS: Die Wehrmacht-Untersuchungsstelle. 1984.

Wehrmachtführungsstab, Abteilung Wehrmachtpropaganda

in Best. RW 4 Die Akten des Bestandes, der auch eine Ausarbeitung des Amtschefs Generalmajor Hasso v. Wedel über seine Tätigkeit (4 Bde, 1957/58, dazu Ergänzung im IfZ, Best. ZS $180)$ enthält, betreffen folgende Gebiete:

- Organisation (12 Bde, 1937-1945), Personalangelegenheiten (13 Bde, 1939-1944) und Inneren Dienst (15 Bde, 1939-1943);

- Propagandaführung im allgemeinen (37 Bde, 1937-1945) und Einsatz der Propagandatruppen (71 Bde, 1939-1944, vor allem bei der Kriegsmarine und in besetzten Gebieten, mit Tätigkeitsberichten);

- Zusammenarbeit mit zivilen Behörden und der Presse (13 Bde, 1938-1942);

- Rundfunk- (7 Bde, 1939-1944), Bild- und Filmpropaganda (11 Bde, 1939-1941);

- Sammlung von Nachrichten über die Lage in der Wehrmacht und im Inland (11 Bde, 1939-1942) und Ausland (29 Bde, 1939-1942);

- Abwehr der Feindpropaganda (10 Bde, 1939-1945, mit Flugblättern);

- Ausübung der militärischen Zensur (89 Bde, 1939-1944, hauptsächlich über Einzelfälle der Prüfung, Genehmigung oder des Verbots von Büchern, Zeitschriften- und Zeitungsartikeln und der Veröffentlichung von Bildern).

Vorhanden sind ferner Serien (insgesamt 26 Bde, 1939-1945) u. a. von WehrmachtPropaganda-Lageberichten, „Nachrichten des $\mathrm{OKW}$ “, „Mitteilungen für das Offizierkorps“, „Mitteilungen für die Truppe“. Die Tätigkeit der Wehrmacht bei der und für die Propaganda ist ferner dokumentiert durch Negative der Aufnahmen von Bildberichterstattern der Propagandakompanien (ca. 1 Million, BA, Bildarchiv), Reste von Rohund Schnittmaterial für die Kriegswochenschauen, von der Heeresfilmstelle und der reichseigenen Mars-Film GmbH hergestellte Filme (u. a. 13 Ausgaben einer „Frontschau“, Lehrfilme wie „Kriegssanitätsdienst", sämtlich BA-Filmarchiv), Plakate (BA, Plakatsammlung) und Flugblätter (BA, ZSg 2).

Lit.: Die WEHRMACHT. 5 Bde (1939-1944). 1978. - E. MURAWSKI: Der deutsche Wehrmachtbericht 1939-1945. 1962. - H. v. WEDEL: Die Propagandatruppen der Deutschen Wehrmacht. 1962. - W. STANG: Organe und Mittel der militärischen Führung des faschistischen Deutschlands zur Meinungsmanipulierung besonders der Soldaten des Heeres 1939-1945. 1980.

Amt Ausland/Abwehr

Best. RW 5

Mit Vorakten der Abwehrabteilung des Reichskriegsministeriums und der Auslandabteilung des Wehrmachtführungsamtes enthält der Bestand (insgesamt ca. $450 \mathrm{Bde}$ ) Schriftgut aus der Zeit, in der Admiral Canaris den militärischen Auslandnachrichtendienst leitete (1935 bis zur Überführung in das Amt Mil des Reichssicherheitshauptamtes im Februar 1944, Auskünfte seines Vorgängers, Admiral Patzig, im IfZ, Best. ZS 540), von folgenden Abteilungen:

Zentralabteilung: Geschäftsverteilungspläne u. a. Organisationsunterlagen (1935-1944, auch für nachgeordnete Stellen), Besoldungs- und Laufbahnvorschriften, Erkennungsmarkenverzeichnisse, einzelne Personalunterlagen, insbesondere von V-Leuten (19391945), Akten in Personal- und Ausbildungs- sowie Haushaltsangelegenheiten (u. a. Bereitstellung von Devisen für Einsatz im Ausland 1935-1944), sonstige Dienststellenverwaltung. 
Amtsgruppe Ausland: Aktenserien über AuBen-, Wirtschafts- und Wehrpolitik einzelner Länder und Ländergruppen (ca. 150 Bde, 1934-1944), Berichte des Aufklärungsausschusses Hamburg-Bremen (vgl. Abschnitt 4.6.4) über einzelne Länder (ca. 90 Bde, 1939-1945), Akten über Behandlung deutscher Kriegsgefangener und Internierter (1939. 1943), Völkerrecht und -verletzungen (1939-1944), Zusammenarbeit mit dem Roten Kreuz (1939-1942), Seekrieg (1939-1942), Gaskriegsvorbereitungen im Ausland und Gasabwehr (1933-1943), Abrüstungsfragen (1934-1935), Pressemeldungen über deutsche VerstöBe gegen den Versailler Vertrag (1933-1935).

Amtsgruppe Abwehr II (Sabotage und Zersetzung): Kriegstagebuch des Gruppenleiters Generalmajor Erwin Lahousen (3 Bde, 1939-1943, mit Aufzeichnungen über einzelne Aktionen, Nachkriegsaufzeichnungen im IfZ, Best. ZS 658), Ausbildungsunterlagen (1939-1944), Schriftwechsel mit Abwehrstellen in Wehrkreisen (1934-1939, vor allem über Personaleinsatz), Vorgänge über V-Leute und einzelne Unternehmungen (Sprengung der Eisenbahnstrecke Genf-Annecy 1940-1941, Unterstützung von Kurden 1942-1943).

Amtsgruppe Abwehr III (Spionageabwehr und Gegenspionage): Sammlung von Geheimerlassen (3 Bde, 1935-1940), Fahndungslisten (1940), Akten über Geheimschutz, auch in der Wirtschaft (1933-1945), Überwachung von Ausländern, auch Kriegsgefangenen, vor allem durch Postkontrolle, Unterlagen über feindliche Agentenschulen (1943-1944).

Für die nicht überlieferten Akten der Amtsgruppe I Nachrichtenbeschaffung, Spionage) bieten wenige Akten von Abwehrstellen (unten 8.4.1.6) Ersatz, Ergänzungsüberlieferung liegt in den Nachlässen von Vizeadmiral Bürkner, Leiter der Gruppe Ausland (Best. N 565) und Oberstleutnant Groscurth (Amtsgruppe II, Best. N 104, weiterer Teil im IfZ), in einer Materialsammlung über die Völkerrechtsgruppe (IfZ, ZS 8/18) sowie einigen Bänden des zu Sabotageeinsätzen bestimmten Lehrregiments Kurfürst (in Best. RW 49) vor.

Lit: H. GROSCURTH: Tagebücher eines Abwehroffiziers. 1970. - M. Graf SOLTIKOW: Ich war mitten drin. 1980. - G. van ROON: Graf Moltke als Völkerrechter im OKW. 1970. - H. HÖHNE: Canaris. 1976.

Allgemeines Wehrmachtamt

Best. RW 6

Der Hauptteil der Überlieferung (insgesamt ca. 450 Bde) stammt aus der für die Beziehungen zur zivilen Verwaltung und der NSDAP zuständigen Abteilung Inland. Dazu gehören Akten über innere Sicherheit (dabei Verbot von Vereinen und Filmen), Verhältnis zur NSDAP (u. a. Differenzen über Wehrpolitik 1933-1937, Verfahren gegen Soldaten vor Parteigerichten 1942-1943, Behandlung jüdischer Frontkämpfer 1933-1938), Abrüstung (1933-1936), Völkerrecht (1940-1941), Verkehr mit dem Auswärtigen Amt (u. a. Einsetzung von Militär- und Marineattachés 1933-1938), innenpolitische Lage und AnschluB Österreichs (1934-1939), Landesverteidigung und Raumplanung (19351938), politische Beurteilung und Überwachung von Wehrmachtangehörigen (19361938), Strafverfahren gegen Offiziere (Einzelfälle, 1935-1938), Kriegskriminalstatistik (1940-1942), Manöver und Übungen (1933-1939), Aufbau der Luftwaffe und Luftschutz (1933-1943), wehrgeistige Führung und nationalpolitische Erziehung (1933-1944, ebenfalls dokumentiert durch 88 zwischen 1939 und 1943 veröffentlichte „Tornisterschriften“ und fünf Jahrgänge „Soldatenblätter für Feier und Freizeit“, 1940-1944), Presse- und Rundfunkangelegenheiten.

Weitere Akten sind vorhanden aus der Wehrmachtfürsorge- und Versorgungsabteilung (u. a. zahlreiche Erlasse, Entwicklung des Versorgungsrechts, Unterbringung von 
Parteigenossen in der Wehrmachtverwaltung, Beteiligung der Wehrmacht am Winterhilfswerk, Verwaltung des Großen Militärwaisenhauses Potsdam, des Invalidenhauses Berlin und anderer Stiftungen), der Abteilung Wehrmachtverlustwesen (Vorschriftensammlung, Statistiken, Organisation des Gräberdienstes, Planung von Ehrenmalen) und vom Chef des Kriegsgefangenenwesens (Heeresdruckvorschrift H.Dv. 38 und andere allgemeine Dienstanweisungen und Merkblätter, 4 Bde mit Statistiken 1941-1945, Vorgänge über Arbeitseinsatz, Postverkehr, italienische Militärinternierte).

Nur geringen Umfang haben Akten der Wehrmachtverwaltungsabteilung (über Ersatz von Kriegsschäden, Reise- und Zahlungsverkehr) und des vom Chef des Amtes selbst geleiteten NS-Führungsstabes (über Aufstellung, Schulung und Einsatz der NSFührungsoffiziere mit Schulungs- und Propagandamaterial, Lage in Westdeutschland im Februar/März 1945); sie fehlen von den Abteilungen Wissenschaft und für Wehrmachtfachschulunterricht wie auch vom Bevollmächtigten für Siedlungsfragen.

Ergänzungsüberlieferung enthalten die Nachlässe von General Reinecke (Best. N 356) und seines Stabschefs Generalmajor Kurt Linde (N 133); Reinecke wurde in Nürnberg im OKW-ProzeB, der letzte Chef des Kriegsgefangenenwesens, SSObergruppenfuhrer Berger, im WilhelmstraBenprozeB verurteilt.

Lit.: TRIALS OF WAR CRIMINALS. vol. 10-14. - R. KÜBLER: Chef KGW. 1984. - W. BESSON: Zur Geschichte des Nationalsozialistischen Führungsoffiziers. 1961. - A. W. ZOEPF: Wehrmacht zwischen Tradition und Ideologie. 1988.

Wehrwirtschafts- und Rüstungsamt

BA-MA, Best. RW 19 Mit Vorakten des Wehrwirtschaftsstabes (ab 1935) und Nachakten des Wehrwirtschaftsamtes bzw. Feldwirtschaftsamtes aus der Zeit nach Eingliederung des Rüstungsamtes in das Reichsministerium für Rüstung und Kriegsproduktion und Ausscheiden des Amtschefs General Thomas 1942 dokumentient der Bestand (ca. 5000 Bde, 1935-1945, davon 23 Bde Wehrwirtschaftliche bzw. Kriegswirtschaftliche Lageberichte 1934-1945, 289 Bde Kriegstagebücher) weite Bereiche der wirtschaftlichen Kriegsvorbereitungen, der Ausstattung der Truppe mit Waffen und Munition, des Arbeitseinsatzes, Transportwesens und der Ausbeutung besetzter Gebiete, aber auch über die Auswirkungen des Luftkriegs. Einen erheblichen Teil bilden Aktenserien über einzelne Länder (ca. $1600 \mathrm{Bde}$ ) und - z. T. ebenfalls länderbezogene - Ausarbeitungen wirtschaftswissenschaftlicher Institute (ca. $1620 \mathrm{Bde}$, u. a Deutsches Institut für Wirtschaftsforschung, Forschungsstelle für Wehrwirtschaft, Institut für Weltwirtschaft, Institut für Konjunkturforschung, Reichsstelle für Bodenforschung, Volkswirtschaftliche Abteilung der I.G. Farbenindustrie). Ergänzende Unterlagen enthalten Akten des Verbindungsoffiziers zum Reichswirtschaftsministerium und anderer nachgeordneter Dienststellen mit zentraler (Best. RW 45) und regionaler Zuständigkeit (Bestände RW 22 bis 32, vgl. auch unten 8.4.1.2).

Lit: G. THOMAS: Grundlagen für eine Geschichte der deutschen Wehr- und Rüstungswirschaft (1944). 1966. - C. BUCHHEIM: Die besetzren Länder im Dienste der deutschen Kriegswirsschaft während des Zweiten Welukrieges. 1986.

\subsubsection{Zentrale Dienststellen und Einrichtungen unter dem Oberkommando der Wehrmacht}

Zu den zahlreichen Dienststellen, die für die gesamte Wehrmacht zuständig waren und von denen Reste von Schriftgut überliefert sind, gehören folgende, deren Tätigkeit auch den zivilen Bereich berührte: 


\subsubsection{Evangelischer und katholischer Feldbischof}

BA-MA, Best. RW 12 I und II

Die wenigen erhaltenen Akten (20 Bde, 1932-1945) vor allem des Evangelischen Feldbischofs Franz Dohrmann (von ihm auch TeilnachlaB N 282 mit Dokumentation seiner Tätigkeit) enthalten Schriftwechsel mit dem Oberbefehlshaber des Heeres (1932-1939), Richtlinien für die Seelsorge (u. a. im Ersatzheer, Lazaretten und Standortkirchen), einzelne Ansprachen und betreffen organisatorische Angelegenheiten, die Betreuung von deutschen Kriegsgefangenen (1939-1940) und zum Tode Verurteilten (1942-1943) sowie die Verteilung religiösen Schrifttums (1939-1942). Ergänzungsüberlieferung enthaiten das „Verordnungsblatt des Evangelischen" (10 Nrn der Jahrgänge 1-4, 1941-1944, RWD 13) bzw. „des Katholischen Feldbischofs der Wehrmacht“" (44 Nm der Jahrgänge 3-9. 1939-1945, ebda) und das „Mitteilungsblatt für die evangelische Wehrmachtsseelsorge“" (8 Nrn der Jahrgänge 1-4, 1936-1939) bzw. „für die evangelische Wehrmachtsgeistlichkeit“ (7 Nrn der Jahrgänge 1-3, 1942-1944).

Lit: H. KUNST (Hrsg.): Gou läBt sich nicht spotten. 1983. - D. BEESE: Kirche im Krieg. 1990. - H. APOLD: Feldbischof Franz Justus Rarkowski im Spiegel seiner Hinenbriefe. 1978.

\subsubsection{Sonderbeauftragter für die Überprüfung des zweckmäßigen Kriegseinsatzes $\quad$ BA-MA, Best. RW 42}

Bei den Erhebungen über Personal, das bei Reichsministerien, obersten und oberen Reichs- und einigen preuBischen Zentralbehörden entbehrlich war und für den Einsatz bei der Wehrmacht oder in der Rüstung in Betracht kam, durch Generalleutnant von Unruh und seine Mitarbeiter ist eine Sammlung von Organisations- und Geschäftsverteilungsplänen sowie Personallisten entstanden, die auch zahlreiche Behörden und Ämter betrifft, von denen Schriftgut sonst nicht überliefert ist (27 Bde, meist Anfang 1943). Der Bestand enthält außerdem noch einige Akten u. a. über Ordensverleihungen.

\subsubsection{Wehrmachtauskunftstelle für Kriegerverluste und Kriegsgefan- gene (WASt)}

Die seit Kriegsbeginn entstandenen Unterlagen, von denen einige sich bis 1990 in der ehemaligen DDR befanden, werden mit weiterem personenbezogenem Schriftgut über ehemalige Angehörige der Wehrmacht und von ihr beschäftigte Zivilisten von der Deutschen Dienststelle (WASt) für die Benachrichtigung der nächsten Angehörigen der ehemaligen deutschen Wehrmacht in Berlin verwaltet. Es handelt sich dabei vor allem um die regelmäBigen Meldungen der Truppenteile über die Ausgabe von Erkennungsmarken an einzelne Soldaten und deren Veränderungen (ca. 100 Millionen Meldungen für Angehörige von Heer und Luftwaffe, 1,145 Millionen für Kriegsmarine) mit Angaben über ca. 20 Millionen Personen, ferner um Verlustmeldungen (150 Millionen), Mitteilungen über Kriegsgefangene in westlichen Gewahrsamsländern und über dort Verstorbene, Karteien und Listen über Kriegsgräber, jeweils mit ergänzenden Unterlagen aus der Nachkriegszeit. Sie bildeten die Grundlage für Zusammenstellungen über Gliederung und kriegsgeschichtliche Daten für viele Verbände aller Wehrmachtteile (z. T. auch BA-MA, Best. RW 48, 60 Bde).

Lit.: JAHRESBERICHTE der Deutschen Dienststelle (WASt). $1956 \mathrm{ff}$. 


\subsubsection{Reichsstelle für Landbeschaffung}

BA-MA, Best. RW 43

Neben wenigen Akten der Dienststellenverwaltung und über den Erwerb von Grundstücken für militärische Zwecke ab 1935 enthält der Bestand (ca. 4300 Bde, 1934-1944) Unterlagen über Enteignungen und Entschädigungen in Einzelfällen in Verbindung mit der Errichtung oder Erweiterung von Truppenübungsplätzen, Munitionsanstalten (Muna), Flugplätzen und Kasernenanlagen; sie befanden sich u. a. in Baumholder, Berchtesgaden (Bauten für OKW), Bergen bei Celle, Dachau (Gelände für die SS), Fallersleben (Volkswagenwerk), Hammelburg, Kiel, Meppen, Munster-Nord und -Süd, Salzgitter (Reichswerke „Hermann Göring“), Sennelager, Bad Tölz (SS-Junkerschule), Wahn, Wildflecken und Wilhelmshaven.

\subsubsection{Reichsumsiedlungsgesellschaft (Ruges) BA-MA, Best. RW 52}

AuBer Geschäftsberichten und Akten der Dienststellenverwaltung (35 Bde), über Personalangelegenheiten (106 Bde allgemeines, 2594 Einzelfälle) ist Schriftgut über Vermessung und Kulturbau (226 Bde), über die Landbeschaffung und Ansiedlung für Wehrmachtzwecke enteigneter Landwirte im allgemeinen (135 Bde) und in Einzelfällen (ca. 600 Bde) vor allem in Ostdeutschland und in der Provinz Hannover (u. a. Lathen und andere Orte bei Meppen, Pattensen mit Vorgängen über die Kriegsgefangenen-Stammlager VI B und C) vorhanden, auch über die Zweigstelle Münsingen (Württemberg, ab 1937).

\subsubsection{Oberkommando des Heeres (OKH) BA-MA, Best. RH 1 bis 15}

Die Überlieferung (insgesamt ca. 7500 Bde), die Vorakten des Chefs der Heeresleitung und des Truppenamtes des Reichswehrministeriums einschlieBt, stammt von

Adjutantur (dabei Unterlagen über Aufrüstung und Auslandsbeziehungen), Generalstab (dabei Schriftwechsel des Generalstabschefs General Beck und Tagebuch des Generalstabschefs General Halder 1939-1942) mit

- Zentralabteilung (dabei Akten über Einmarsch in Rheinland, Österreich und Sudetenland, Landesbefestigung und zivile ReichsverteidigungsmaBnahmen),

- Operations- und Organisationsabteilungen (mit Kriegstagebüchern, Unterlagen über Dienstrecht, Militärverwaltung im Reichsgebiet, Einsatz von SS, Polizei und RAD, Rüstungsmaßnahmen),

- Abteilungen Fremde Heere West und Ost (mit nachrichtendienstlichen Erkenntnissen auch über innenpolitische und wirtschaftliche Verhältnisse einzelner Länder einschlieBlich Österreichs vor 1938, verbündeter und neutraler Staaten ab 1933) und

- Heeresausbildungsabteilung (mit Offiziersausbildungsabteilung, dabei Studie über nationalsozialistische Wehrreform um 1944, Unterlagen über Zusammenarbeit mit der Reichsjugendführung).

Die Akten werden ergänzt durch Teile von Nachlässen u. a. der Oberbefehlshaber Frh. v. Hammerstein-Equord und Frh. v. Fritsch, Becks und Halders und des Generalquartiermeisters Wagner. Die Intrige, die 1938 zur Entlassung Fritschs führte, ist durch Aufzeichnungen und Aussagen seines Rechtsbeistands und mehrerer Zeugen dokumentiert (IfZ, ED 77, ED 60, ZS 148 und von Prof. Deutsch gesammeltes Material ZS A 47). Einen gesonderten Bestand (Kart RH 2) bilden die z. T. täglichen Lage- und Dislozienungskarten auch über die Aufstellung der Truppen und den Frontverlauf in Westdeutschland 1939/40 und 1944/45 (ca. 8150 Karten, 1934-1945). Der Stabschef des NS-Führungsstabes im OKH, Generalleutnant Hübner, wurde 1948 vom Landge- 
richt München I wegen seiner Tätigkeit als Vorsitzender eines Standgerichts verurteilt (JUSTIZ UND NS-VERBRECHEN Nr. 103).

Lit: GULES to German Records. vol. 29, 30. - FINDBÜCHER zu Beständen des Bundesarchivs Bd. 33. 1988. - K.J. MÜLLER: General Ludwig Beck. 1982. - F. HALDER: Kriegstagebuch. 3 Bde. 1962-1964. W. ERFURTH: Die Geschichte des deutschen Generalstabes von 1918 bis 1945. 1957. - H.-H. WILHELM: Die Prognosen der Abteilung Fremde Heere Ost 1942-1945. 1975. - U. RINGSDORF: Organisatorische Entwickiung und Aufgaben der Abreilung Fremde Heere Ost im Generalstab des Heeres. 1989. - K.-J. THIES: Der Zweite Welturieg im Karenbild. 5 Bde. $1990 \mathrm{ff}$.

Besonders zu berücksichtigen sind folgende Teilbestände :

Chef der Heeresrüstung und Befehlshaber des Ersatzheeres

Best. RH 14

Die Tätigkeit und Zuständigkeit der Dienststelle u. a. für die Truppen im Reichsgebiet ist nur durch wenige Akten aus der Zeit unter Generaloberst Fromm bis zum 21. Juli 1944 belegt, und von seinem Nachfolger, dem Reichsführer SS Himmler, als Oberbefehlshaber des Ersatzheeres liegt lediglich eine Befehlssammlung (1944-1945), eine Rede vor den Wehrkreisbefehlshabern (September 1944) und Material über Ausbildung und Einsatz des Volkssturms vor, dazu als Ergänzung eine Aufzeichnung des Ingenieurs Werner Kennes (IfZ, Best. ZS 1747). Die Akten (47 Bde) stammen von folgenden Organisationseinheiten:

Stab (seit Oktober 1943 unter Oberst Graf Schenk von Stauffenberg): Kriegstagebuch (1941-1942), Befehle und Gegenbefehle "Walküre" am 20. Juli 1944, Organisationsunterlagen (Geschäftsverteilungspläne 1939-1942, Geheimerlasse 1939-1941, u. a. über MaBnahmen in besetzten Gebieten), Berichte der Truppe zur innenpolitischen Lage und inneren Sicherheit (4 Bde, 1939-1942), Unterlagen über Truppenbetreuung, bevölkerungspolitische Maßnahmen (,Lebensborn“), Bedarf an Rohstoffen und Arbeitskräften (dabei Bestellung von Verbindungsoffizieren zu den Gauleitern 1944), Waffen- und Munitionsbestände, Verleihung von Orden (1940-1944).

Amtsgruppe Heeresrechtswesen: Sammlung grundsätzlicher Erlasse zum Militärstrafrecht (12 Bde, 1937-1945), einzelne Vorgänge über Strafvollzug und Dienstrecht.

Lit: H.-A. KOCH: Gliedenung des Ersatzheeres (Stand etwa Frühjahr 1943). 1957.

Allgemeines Heeresamt

Best. RH 15

Das Schriftgut (450 Bde) ist in folgenden Dienststellen des unter dem Befehlshaber des Ersatzheeres bis zum 20. Juli 1944 von Generalleutnant Friedrich Olbricht geleiteten Amtes entstanden:

Stab: Mobilmachungspläne und -anordnungen (ab 1936), Erfahrungsberichte der Waffenkommissionen (13 Bde, 1940-1941), Akten über Aufstellung, Umgliederung und Auflösung von Dienststellen und Verbänden (Heeresaufbau 1935, Demobilisierungsmaßnahmen 1940, Maßnahmen ab September 1944), personelle und materielle Ausstattung des Heeres, Erprobung von Waffen und Munition, Wehrerziehung durch die SA (1940).

Adjutantur: Stellenbesetzungspläne des Amtes und seiner nachgeordneten Dienststellen, Unterlagen über Personalangelegenheiten (dabei Entlassung jüdischer Mischlinge 1940, katholischer Theologiestudenten und Geistlicher 1944), Disziplin und Ordnung (u. a Einführung des „Deutschen Grußes“ 1944).

Zentralabteilung: Haushaltsunterlagen und Akten über Personalverstärkung, vormilitärische Ausbildung, Tätigkeit des Reichsverteidigungsrates. 
Amtsgruppe Ersatz- und Heerwesen: Sammlung von Erlassen über Erfassung, Musterung und Annahme von Wehrpflichtigen und Freiwilligen (21 Bde, 1935-1945) und von Dienstanweisungen und Merkblättern in Mobilmachungsangelegenheiten (39 Bde, 1938-1943), spezielle Unterlagen über Wehrpflicht ausländischer Minderheiten und in Österreich und anderen eingegliederten Gebieten, über Organisation der Wehrersatzdienststellen, Dienstrecht, auch der Wehrmachtbeamten, Auflösung von Einheiten nach den Kapitulationen in Stalingrad und Tunis.

Amtsgruppe Seelsorge (ca. 20 Bde, 1934-1945): Akten zur Organisation in den Wehrkreisen, Einstellung, Ausrüstung und Besoldung haupt- und nebenamticher Standortpfarrer, allgemeine Richtlinien (auch zum Verhältnis der Kirchen zu Staat und NSDAP). Abwicklungsstab: Unterlagen über die Abwicklung von Angelegenheiten zerschlagener Verbände der 6. Armee in Stalingrad, der Heeresgruppen Mitte, Nord- und Südukraine und des Oberbefehlshabers West (mit Erlebnisberichten von Rückkehrern, Erhebungen über Kriegsgefangene und VermiBte, nachträgliche Beförderungen und Auszeichnungen, mit Einzelfällen).

Die Tätigkeit des Amtes ist außerdem durch die umfangreichen Serien der von dort herausgegebenen Heeresdruckvorschriften (H.Dv.), Merkblätter, Friedens- und Kriegsstärke- und -ausrüstungsnachweisungen, das "Jahrbuch des deutschen Heeres“ (1936-1942), die ,Zeitschrift für die Heeresverwaltung“ (1936-1944) und das „Heerestechnische Verordnungsblatt“ (ab 1943) dokumentiert.

Lit.: F. GEORGI: Soldat im Widerstand. General der Infanterie Friedrich Olbricht. 1988.

Heerespersonalamt

Best. RH 7

Überliefert sind einerseits Dienstalters-, Stellenbesetzungs- und andere Listen mit entsprechendem Schriftwechsel und die chronologischen Serien der Mitteilungen über Beförderung und Versetzung von Offizieren (1934-1945) sowie Sachakten u. a. über die Erziehung und Ehrauffassung des Offizierkorps, andererseits Personalakten von 257 Generalen in Spitzenstellungen und von 66635 weiteren Offizieren (überwiegend noch bei der AuBenstelle ZNS des BA, dort auBerdem ca. 400000 Karteikarten über Offiziere auch der Reserve dieser und zahlreiche Personalakten anderer Provenienz). Ein weiterer Teilbestand enthält (überwiegend ebenfalls bei ZNS) Akten, Karteien und Listen über die Verleihung von Orden und Auszeichnungen an Angehörige des Heeres und der Waffen-SS und ihres zivilen Gefolges (ca. 5,5 Millionen Einzelfälle), dabei über sämtliche Stufen des Eisernen Kreuzes und des Kriegsverdienstkreuzes mit und ohne Schwerter wie auch über Verleihung ausländischer Orden an Offiziere (2 700 Blatt ab 1937).

Lit.: TÄTIGKEITSBERICHT des Chefs des Heerespersonalamtes 1942-1944. 1984. - RANGLISTE des Deutschen Heeres 1944/45. Hrsg. von W. Keilig. 1955. - R. STUMPF: Die Wehrmachts-Elite. 1982

Weitere Teilprovenienzen, auf die hier nur verwiesen werden kann, sind der Generalquartiermeister, die Chefs des Transport-, Kriegskarten- und Vermessungs- und Heeresnachrichtenwesens, der Generalinspekteur der Panzertruppen und die Generale für einzelne Waffengattungen, das Heeresverwaltungs- und Heereswaffenamt.

Lit: E. WAGNER: Der Generalquartiermeister. 1963. - E. LEEB: Aus der Rüstung des Dritten Reiches (Das Heereswaffenamt 1938-1945). 1958. 


\subsubsection{Oberkommando der Kriegsmarine (OKM)}

BA-MA, Best. RM 6 und 7

Den Hauptteil der Überlieferung aus der Zeit nach 1933 bildet das Kriegstagebuch der Seekriegsleitung mit seinen Anlagen (1 580 Bde, 1939-1945), das auch politische Übersichten (21 Bde, 1939-Juli 1943) und Lageübersichten über Politik, Völkerrecht, Propaganda (28 Bde, 1939-1945) und Handelsschiffahrt (6 Bde) enthält. Relativ umfangreich sind außerdem Akten der Referate für Völker- und Seekriegsrecht (ca. 130 Bde, 1937-1945, u. a. betr. Neutrale, Prisenrecht, Lazarettschiffe, Zwischenfalle mit den USA 1940, Hilfsaktionen für die Zivilbevölkenung in besetzten Gebieten), des Generalreferats für Rüstung (ca. 50 Bde, 1934-1944), der Gruppe Handelsschiffahrt und Wirtschaftskrieg, des Marinenachrichtendienstes über Schiffsbewegungen im Spanischen Bürgerkrieg und im Krieg, ferner Unterlagen über die japanische Marine (7 Bde, 1934-1938) und die Zusammenarbeit mit der Sowjetunion 1939-1941, mit Italien und Spanien (20 Bde, 1939-1943) sowie über Propaganda (ca. 30 Bde, 1939-1943, dabei Sprachregelungen, Artikeldienst und Vorträge, vor allem von Konteradmiral Lützow). Ergänzungsüberlieferung enthalten die Dokumente des IMT-Verfahrens gegen die Oberbefehlshaber und GroBadmirale Raeder und Dönitz und deren Nachlässe (N 391, N 236) und gegen den Flottenchef Generaladmiral Schniewind im Nürnberger OKW-ProzeB, außerdem der NachlaB des Generaladmirals Boehm (N 172) und Material des Admirals Förste (N 328).

Lit.: GUIDES to German Records. vol. 37. - KRIEGSTAGEBUCH der Seekriegsleitung 1939-1945. 1988 ff. - M. SALEWSKI: Die deutsche Seekriegsleitung 1935-1945. 3 Bde. 1970-1975. - E. RAEDER: Mein Leben. 1956/57. - Karl DÖNITZ: Zehn Jahre und zwanzig Tage. 1958.

\subsubsection{Zentrale Dienststellen der Kriegsmarine}

Aus der Überlieferung der zahlreichen Hauptämter, Ämter, Inspektionen, Kommissionen und sonstigen Kriegsmarinedienststellen, die dem Oberkommando direkt unterstanden und sich u. a. mit dem Bau und der Ausrüstung der Schiffe und der Verwaltung der Häfen und Werften befaßten, ist folgendes anzuführen:

- Verwendungskartei und Personalakten der Admirale (BA-MA, Best. RM 17) und von ca. 72000 Marineoffizieren und Offiziersanwärtern aus dem Marinepersonalamt (Deutsche Dienststelle Berlin).

- Akten des Marineverwaltungsamtes (BA-MA, Best. RM 23) über die Beschaffung von Rohstoffen und Lebensmitteln (75 Bde, 1934-1941).

- Akten des Marinekommandoamtes (BA-MA, Best. RM 20) über die Aufrüstung (15 Bde, 1933-1939) und über Vorbereitung, Verlauf, politische, wirtschaftliche und militärische Aspekte der Beteiligung am Spanischen Bürgerkrieg (334 Bde, 1936-1939). - Unterlagen des Wehrgeistigen Führungsstabes im Allgemeinen Marinehauptamt (1943-1945, BA-MA, Best. RM 22 mit Ergänzungsüberlieferung im Nachlaß N 599 des Fregattenkapitäns Frühling).

- Filme der Marine-Hauptfilm- und Bildstelle (19, 1938-1944, BA-Filmarchiv). Von Bedeutung für die Geschichte der Technik sind wenige Akten und zahlreiche Pläne (BA-MA, Best. RM 25) aus dem Hauptamt Kriegsschiffbau, dessen Überlieferung sich vor allem im ehem. Militärarchiv der DDR befindet (21 000 Bde, 1935-1945); sie dokumentiert nahezu alle Schiffsklassen wie Schlachtschiffe, Kreuzer, Zerstörer. U-Boote, Minenschiffe und die Zusammenarbeit mit zivilen Werften auch in den besetzten Westgebieten, Norwegen und Dänemark mit einzelnen Akten über Ausbildung 
von Nachwuchs, zur Planung des Kräftebedarfs und zum Einsatz von Arbeitskräften, darunter Frauen und Kriegsgefangene, im Schiffbau.

Zum Reichskommissar beim Prisenhof Hamburg vgl. Abschnitt 3.2.4.6.

\subsubsection{Reichsministerium der Luftfahrt und Oberkommando der Luftwaffe (OKL) BA-MA, Best. RL 1 bis 6}

Die sehr unvollständige Überlieferung, die nur ganz wenig aus der 1933 vom Reichsverkehrsministerium übernommenen Zuständigkeit für den zivilen Luftverkehr enthält, besteht aus einigen Vorgängen aus der Umgebung Görings (27 Bde, dabei Reden, Aufrufe und Erlasse, vor allem zu Disziplin und Rechtspflege, Organisation und Rüstung), Akten des Generalstabs der Luftwaffe mit Luftwaffenführungsstab (dabei 7 Bde betr. Luftschutz, 1939-1945, 4 Bde betr. Krisenmaßnahmen, 1944-1945), Generalquartiermeister (mit umfangreichen Unterlagen über Organisation der Luftwaffe auch im Reichsgebiet und Lieferungen der Rüstungsindustrie $u$. a. an befreundete und neutrale Staaten), Kriegswissenschaftlicher Abteilung (dabei einzelne Studien über den Luftkrieg gegen Deutschland) und Generalnachrichtenführer (mit Angaben über Luftangriffe 1944/45), des Chefs des Ausbildungswesens und der Generale für bestimmte Waffengattungen (mit Erfahrungsberichten iiber schwere Luftangriffe auf deutsche Städte).

Als Ergänzungsüberlieferung ist auf Dokumente aus dem IMT-ProzeB gegen Göring und das amerikanische Verfahren gegen den Staatssekretär der Luftfahrt und Generalinspekteur der Luftwaffe Generalfeldmarschall Milch und dessen Nachlaß (Best. N 179), die Handakten und das persönliche Kriegstagebuch des Chefs des Generalstabs General Werner Kreipe (ab 1943) in dessen Nachlaß (Best. N 141), ferner auf Aufzeichnungen des Leiters der Rechtsabteilung, Ministerialdirektor Christian v. Hammerstein (IfZ, Best. ED 8) zu verweisen. Beachtliches Quellenmaterial über die deutsche Luftrüstung ab 1933 und den Einsatz der Luftwaffe im Zweiten Weltkrieg an den Fronten und zur Verteidigung des Luftraums über Deutschland enthält die für die USA von Luftwaffenoffizieren nach 1945 angelegte "Sammlung Rhoden“ (BA-MA, Mikrofilm IfZ, MA 54/1-3). Der zivile Luftschutz wird vielfach in Schriftgut der allgemeinen inneren Verwaltung in Reichsverteidigungs- (vgl. Abschnitt 2.1) und Bauangelegenheiten (vgl. Abschnitt 5.6) dokumentiert und, z. B. im ZStA, in Spezialinventaren nachgewiesen.

Die Überlieferung folgender Organisationseinheiten ist wegen eines gröBeren Anteils an Schriftgut zur Wirtschafts-, Technik- und Landesgeschichte von Bedeutung:

Generalluftzeugmeister

BA-MA, Best. RL 3 $\mathrm{Zu}$ dem umfangreichen Bestand (ca. $2250 \mathrm{Bde}$ ), in dem sich auch wichtige Unterlagen über die Rüstungsproduktion in den letzten Kriegsjahren (u. a. über Konferenzen Görings und Milchs mit dem Reichsminister für Rüstung und Kriegsproduktion und des beiden Ressorts gemeinsamen Jägerstabes, Protokolle von Wochenbesprechungen auch IfZ, Best. ED 315) befinden, gehört vor allem Korrespondenz schon aus der Vorkriegszeit mit Firmen der Flugzeugindustrie wie Arado, Dornier, Fieseler, Focke-Wulf, Heinkel, Henschel, Junkers, Klemm und Messerschmidt und anderen Lieferanten der Luftwaffe, z. B. AEG, BMW, Blohm und Voss, Daimler-Benz und Siemens, ferner mit dem Reichsforschungsrat und Material über Arbeitseinsatz und Lieferungen aus besetzten Gebieten, auch über die Entwicklung der Strahltriebwerke. 
Luftwaffenpersonalamt

BA-MA, Best. RL 5

Der Bestand enthält neben Aktenresten verschiedener anderer Organisationseinheiten (u. a. über NS-Führungsoffiziere und Erlassen zur Rechtspflege) vor allem Meldungen der Einheiten der Flakartillerie über den AbschuB feindlicher Flugzeuge, von denen etwa $40 \%$ (ca. 200 Bde, 1942-1944) aus dem späteren Gebiet der Bundesrepublik und Berlin stammen. Mit Ausnahme der Personalakten von 122 Generalen (BA-MA, Best. Pers. 6) befinden sich die Akten über ca. 73000 Offiziere, Ingenieure und Beamte der Luftwaffe und umfangreiches weiteres personenbezogenes Schriftgut des Amtes noch bei der AuBenstelle ZNS.

Luftwaffenverwaltungsamt

BA-MA, in Best. RL 6 Die Aktenreste betreffen u. a. bauliche Anlagen in den Luftgauen VI (Münster), VII (München) und XII (Wiesbaden).

\section{Allgemeines Luftamt}

BA-MA, in Best. RL 6

Als einzige Unterlagen aus der Zuständigkeit für die Zivillufffahr sind ein Tätigkeitsbericht für 1943/44 und Akten (27 Bde) über Sperrgebiete für zivilen Flugverkehr 1932/33, über Flugverbindungen mit Japan 1942/43 und Italien und anderen europäischen Ländern im Krieg, die Reichsbeteiligung an Flughafengesellschaften (19421944), Flugsicherung und Unfälle erhalten.

Lil: GUDES to German Records. vol. 13 und 24. - TRLALS of War Criminals. vol. Il. - RANGLISTE der deutschen Luftwaffe vom 20. 4. 1945. 1954. - H. WEISS: Die Aufzeichnungen Hermann Görings im Institut für Zeitgeschichte. 1983. - A. KUBE: Pour le mérite und Hakentreuz 1987. - S. MARTENS: Hermann Göring. 1985. - D. IRVING: Die Tragödie der Deutschen Luftwaffe. 1970. - H. BOOG: Die deutsche Luftwaffenführung 1935-1945. 1982. - K. KOLLER: Der letzte Monat. 1985.

\subsubsection{Zentrale Dienststellen der Luftwaffe}

\subsubsection{Forschungsamt dis Reichsluftfahrtministeriums}

BA, Best. Kl. Erw. 272

Aus der Tätigkeit des Amtes zur Überwachung des privaten und diplomatischen Fernsprech- und Funkverkehrs schon in der Vorkriegszeit ist nichts uberliefert, Berichte über Sendungen ausländischer Rundfunkstationen wurden mit entsprechendem Material anderer Provenienz vereinigt (vgl. Abschnitt 4.6.6). Über ihre Aufgaben haben jedoch der Amtschef Ministerialdirektor Schappers und ehemalige Mitarbeiter 1945 bei Befragungen durch amerikanische Offiziere und in 1951, 1953 und 1958 in Denkschriften berichtet (3 Bde und IfZ ZS 291, 1409, 1734, 2316, auch Fg 36 und Fd 56). Der Bestand enthält nur Reste von Akten der Dienststellenverwaltung (5 Bde, 1943-1945) und Personalunterlagen und -karten über ca. 120 (7 Bde, nur Anfangsbuchstaben C T) bzw. 1200 Amtsangehörige (Mikrofilm, 1941-1943).

Lit: D. IRVING: Das Reich hört mit: Görings „Forschungsamt“. 1989.

\subsubsection{Luftwaffen-Forschungsinstitute BA-MA, Best. RL 39}

Der Bestand vereinigt Unterlagen und Berichte (ca. 180 Bde) vor allem der Deutschen Akademie für Luftfahrtforschung, Berlin und München, der Technischen Akademie der Luftwaffe, Berlin-Gatow, und ihrer Institute, der Aerodynamischen Versuchsanstalt Göttingen, der Deutschen Versuchsanstalt für Luftfahrt, Berlin-Adlershof, und 
ihrer Institute, der Deutschen Forschungsanstalt für Segelfug „Ernst Udet“, Ainring, der Reichsstelle für Hochfrequenzforschung, des Instituts für Luftfahrtmedizin (dazu Ergänzungsüberlieferung in Akten des Nümberger Verfahrens gegen Mediziner, in dem dessen Direktor Weltz zusammen mit dem Chef des Luftwaffensanitätswesens, Generaloberstabsarzt Oskar Schröder, dem Generalarzt Gerhard Rose und vier weiteren Ärzten und Sanitätsoffizieren der Luftwaffe angeklagt waren) und von einzelnen Instituten an Technischen Hochschulen.

Lit: TRIALS of War Criminals. vol. I und II. - K.-H. EYERMANN: Der grobe Bluff. Aus Geheimarchiven der deutschen Luftfahr. 1963.

\subsubsection{Reichsamt für Wetterdienst}

BA-MA, Best. RL 40

Erhaiten blieben nur eine Sammlung von Vorschriften und Publikationen (57 Bde, 19361944), Korrespondenz mit Klimabeobachtungsstellen (12 Bde, 1941-1945), Aktenreste aus der Dienststellenverwaltung (4 Bde, 1933-1941), ergänzt durch Material aus dem Aeronautischen Observatorium in Lindenberg/Brandenburg (10 Bde) und der Wetterbezirkszentrale Berlin-Tempelhof (in Best. RL 28, 18 Bde, 1937-1945, dabei Wetterberichte und -vorhersagen zur Beratung von Flak und fliegenden Verbänden).

\subsubsection{Hauptfilmstelle des Reichsluftfahrtministeriums}

Von den in ihrem Auftrag und später der Mars-Film GmbH hergestellten Lehrfilmen ist eine Reihe, z. B. „AbschuBfilm Tagjagd“" und „Flieger in Bergnot“ (1944), überliefert (BA-Filmarchiv, 61 Titel, 1939-1944).

\subsection{Militärische Dienststellen in verbündeten und neutralen Staaten}

Die Beziehungen der Wehrmachtführung und der Oberkommandos der Wehrmachtteile zu den Streitkräften anderer Staaten in der Vorkriegs- und Kriegszeit sind auBer durch Akten der Amtsgruppe Ausland des OKW und der Attaché-Abteilungen des OKH (in RH 2, 55 Bde, dabei Kriegstagebuch 1942/43 und Korrespondenz mit fremden Militärattachés in Berlin), OKM (Best. RM 11, ca. 75 Bde, überwiegend betr. einzelne Staaten, dabei 16 Bde über Italien, 14 über Japan, 6 über Sowjetunion 1938-1943) und OKL (Best. RL 2 VI, 6 Bde) durch Aktenreste der Militärattachés und anderer ins Ausland entsandter Offiziere in folgenden Beständen dokumentiert:

\subsubsection{Militärattachés}

Von den Vertretern des Heeres, die in kleineren Ländern auch die Belange von Kriegsmarine und Luftwaffe vertraten, stammen Akten (Best. RH 67) der Militärattachés in London (47 Bde, 1933-1939) und in Rom (44 Bde, 1933-1940, z. T. bis 1942); derselbe Bestand enthält außerdem Korrespondenz der Attachéabteilung mit den Vertretern von 22 Ländern (ca. 70 Bde, 1929-1945). Weitere Unterlagen bieten die Nachlässe der Generale Friedrich v. Boetticher (N 323), Militärattaché in Washington und Mexiko (1933-1942), Ernst Köstring (N 123, 4 Bde, 1935-1940, auch im Nachlaßteil im ZStA, Best. 90 Ko 7), Militärattaché in Moskau (1933-1935) und Leo Frh. Geyr v. Schweppenburg (N 254, weiterer Teil im IfZ, ED 91), Militärattaché in London (1933-1936) 
sowie Nachkriegsaufzeichnungen der Militärattachés in Helsinki, Istanbul und Warschau (in Best. MSg 1 und 2).

Besser überliefert sind Akten und auch Kriegstagebücher der Marineattachés (Best. RM 12) vor allem in London (101 Bde, 1933-1939, mit Privatkorrespondenz von Kapitän z. S. Leopold Siemens), Rom (62 Bde, 1933-1943), Tokio (33 Bde, auch der MarineEtappe Yokohama, 1939-1945), ferner Moskau (15 Bde, 1932-1941), Istanbul bzw. Ankara (9 Bde, 1939-1944), San Sebastian bzw. Madrid (7 Bde, 1939-1944), Lissabon (6 Bde, 1940-1944), Agram (9 Bde des Marineverbindungsstabes) und in den vier skandinavischen Hauptstädten. Die Überlieferung der Luftwaffenattachés (in Best. RL 2 II) beschränkt sich auf wenige Akten der Vertreter in London (30 Bde, 1933-1939) und Brüssel (4 Bde, 1938-1939).

Vom Wehrwirtschafts- und Rüstungsamt im OKW waren Wehrwirtschaftsoffiziere an Auslandsvertretungen abgeordnet, von denen Kriegstagebücher, Lageberichte und einige Akten aus Bern (3 Bde, 1943-1944, auBerdem 5 Bde der Deutschen Industriekommission), Lissabon (6 Bde, 1942-1944), Madrid (3 Bde, 1943-1944) und Stockholm (10 Bde, 1941-1944, mit Ergänzungen im NachlaB von Oberst Curt Drews, N 461) vorliegen (in Best. RW 45).

Lit: L. Frh. GEYR v. SCHWEPPENBURG: Erinnerungen eines Militärattachés. 1949. - H. TESKE: General Ernst Köstring. 1965.

\subsubsection{Militärmissionen und Bevollmächtigte Generale}

BA-MA, Best. RH 31

Zur Vertretung der Interessen der Wehrmacht in verbündeten Staaten wurden dort Dienststellen errichtet, deren Leiter vor allem in den letzten Kriegsjahren auf die Regierungspolitik der Länder, die faktisch von der Wehrmacht besetzt waren, starken Einfluß ausübten. Folgende Bestände sind vorhanden:

Deutsche Heeresmission in Rumänien, ab 1943 Deutscher General beim Oberkommando der rumänischen Wehrmacht (225 Bde, 1940-1944).

Deutscher Bevollmächtigter General in Kroatien (17 Bde, 1941-1945).

Bevollmächtigter General der deutschen Wehrmacht in Italien (45 Bde, 1943-1945. Ergänzungsüberlieferung in Akten des in Personalunion verbundenen Höchsten SS- und Polizeiführers Italien im BA, Best. R 70 Italien).

Lit.: GUIDES to German records. vol. 5. 18 und 38. - G. FRICKE: Kroatien 1941-1944. 1972.

\subsection{Oberkommandos und Generalkommandos}

Von der Überlieferung der Kommandobehörden von Heeresgruppen und Armeen und der entsprechenden Großverbände von Luftwaffe und Kriegsmarine ist für die allgemeine und die Landesgeschichte diejenige bedeutsam, die vor dem Krieg oder bei Kampfhandlungen und deren Vorbereitung im Reichsgebiet entstanden ist. Auf sie beschränkt sich die nachfolgende Übersicht. Ergänzungsüberlieferung liegt über die Befestigungen im Westen (Best. MSg 100) und über die Kämpfe im Hürtgenwald bei Aachen 1944 (12 Bde, MSg 172) vor. 
Die Bestände enthalten hauptsächlich Kriegstagebücher (KTB) und Tätigkeitsberichte, in denen regelmäßig alle wesentlichen militärischen Handlungen und Ereignisse zu verzeichnen waren, und zum Teil auch Anlagen dazu, in denen u. a. Befehle und Meldungen gesammelt wurden. Sie wurden abteilungsweise geführt. Unter dem Chef des Stabes standen in der Führungsabteilung der Erste Generalstabsoffizier (la), der für die Truppenführung zuständig war, und der für Feindaufklärung und Abwehr, aber auch geistige Betreuung und Propaganda verantwortliche Dritte Generalstabsoffizier (Ic). Dem Leiter der Quartier- (bzw. Oberquartier-) meisterabteilung und Zweitem Generalstabsoffizier (Ib oder OQu) oblag die Versorgung der Truppe mit Waffen, Munition und Ausrüstungsgegenständen. Personalangelegenheiten der Offiziere bearbeitete der Erste Adjutant (Mla), der Unteroffiziere und Mannschaften der Zweite (IIb). Der Intendant als Leiter der Verwaltung und des Rechnungswesens führte die Bezeichnung IVa, der leitende Sanitätsoffizier IVb, die beiden Geistlichen firmierten als IVd. Weiteren Stabsoffizieren waren Ausbildungs- (Id). Transport-, Wehrwirtschafts- und Pionierangelegenheiten, Nachrichtenverbindungswesen und Einsatz der Luftwaffe (Koluft) übertragen. III bezeichnete den Gerichtsoffizier (vgl. dazu Abschnitt 3.2.2.3).

\subsubsection{Oberkommandos von Heeresgruppen BA-MA, Best. RH 19}

Im späteren Gebiet der Bundesrepublik Deutschland waren vor Kriegsausbruch die Heeresgruppenkommandos 2 in Frankfurt/Main und 6 in Hannover zuständig. Während vom Heeresgruppenkommando 6 wie auch vom Berliner Heeresgruppenkommando 1 nur Aktensplitter überliefert sind ( 2 bzw. 4 Bde, Best. RH 64), liegen vom Heeresgruppenkommando 2 umfangreichere Akten als Vorakten des daraus gebildeten Oberkommandos der Heeresgruppe $C$ vor. Sie betreffen die Herbstmanöver 1936 (3 Bde), den Bau der Westbefestigungen (49 Bde, 1938-1940, dabei 4 Bde betr. RAD) und den Aufmarsch an der Westfront und seine Vorbereitung (27 Bde, 1936-1939); sie werden ergänzt durch Aufzeichnungen des Oberbefehlshabers Generaloberst Wilhelm Adam (IfZ, ED 109).

Aus den an der Westfront und bei der Vorbereitung des Überfalls auf Belgien und die Niederlande zwischen September/Oktober 1939 und dem 10. Mai 1940 eingesetzten Oberkommandos von Heeresgruppen ist vor allem folgende Überlieferung vorhanden: Heeresgruppe A (Eifel, Hunsrück): Chef des Stabes (3 Bde), Ia (51 Bde), Ib (4 Bde), OQu (19 Bde).

Heeresgruppe B (Raum Aachen, Niederrhein): Ia (ca. 60 Bde), Ic (20 Bde).

Heeresgruppe $C$ (zunächst ganze Westgrenze, ab Oktober 1939 Pfalz und Oberrheingebiet): Ia (ca. 150 Bde einschließlich der Vorakten), Ic (33 Bde), OQu (11 Bde), Ergänzungen im Nachlaß des Oberbefehlshabers Generaloberst Wilhelm Ritter von Leeb (N 145).

Sehr viel unvollständiger ist die Überlieferung aus den Kämpfen im Westen 1944/45. Sie beschränkt sich auf folgende Teilbestände:

Heeresgruppe B (Eifel, Hunsrück): Ia (5 Bde), Ic (6 Bde), außerdem jedoch Akten aus 36 Standgerichtsverfahren (1944-1945).

Heeresgruppe D und Oberbefehlshaber West (für die ganze Front): Tagesbefehle, Akten des Chefs des Stabes über die Ardennenoffensive (6 Bde), Ia (30 Bde), Ic (7 Bde), NS-Führungsoffizier (2 Bde).

Heeresgruppe G (Pfalz, Oberrheinfront): Ia (20 Bde), Ic (3 Bde), OQu (15 Bde, u. a. betr. Zerstörung kriegswichtiger Anlagen vor einem Rückzug, ARLZ-Maßnahmen).

Von den Heeresgruppen $\mathbf{H}$ (Aachen, Niederrhein) und (nur kurze Zeit bestehend) Oberrhein sind lediglich Aktensplitter ( 4 bzw. 2 Bde) vorhanden. 
Lit.: GUIDES to German Records. vol. 40 und 52. - A. HOCH. H. WEISS: Die Erinnerungen des Generalobersten Wilhelm Adam. 1980. - G. MEYER: Generalfeldmarschall Wilhelm Ritter von Leeb. 1976.

\subsubsection{Oberkommandos von Armeen}

BA-MA, Best. RH 20

Von den $1939 \mathrm{im}$ Westen aufgestellten oder nach dem Polenfeldzug dorthin verlegten Armeeoberkommandos (AOK) sind aus der Zeit vor dem 10. Mai 1940 folgende Bestände vorhanden, die durch die Überlieferung der ihnen jeweils unterstellten Generalkommandos von Armee- und Panzerkorps (Nachweis bei G. TESSIN, Verbände und Truppen) und der zu ihnen gehörenden Divisionen ergänzt werden (Bestände RH 24, 26, 27):

AOK 1 (Saargebiet, Pfalz): Ia (27 Bde), Ic (2 Bde), OQu (19 Bde), IVa (13 Bde), IVb (9 Bde), IVd (3 Bde).

AOK 4 (Raum Aachen): Ia (25 Bde), Ic (1! Bde), OQu (32 Bde), IVa (5 Bde), Gruppe 570 der Geheimen Feldpolizei (4 Bde).

AOK 5 (Eifel, nur bis Mitte Oktober 1939): Ia (10 Bde), OQu (14 Bde).

AOK 6 (Raum Kleve): Ia (32 Bde), lc (3 Bde), OQu (8 Bde), IVa (8 Bde).

AOK 7 (Obenthein): Ia (3 Bde), Ic (12 Bde), OQu (16 Bde), IVa (20 Bde), IVb (6 Bde). AOK 12 (Eifel): Ia (18 Bde), Ic (9 Bde), OQu (ca. 30 Bde).

AOK 18 (niederländische Grenze): Ia (17 Bde), Ic (5 Bde), OQu (12 Bde).

Oberkommando der Armeeabteilung A (niederländische Grenze, nur bis Anfang OKtober 1939): Ia (2 Bde KTB), Ic (8 Bde, dabei Meldungen der Sicherheitspolizei).

Die Kämpfe 1944/45 sind vor allem durch KTB und Akten des AOK 19 (Oberrheinfront, Südwestdeutschland) dokumentiert: Chef des Stabes (4 Bde, 1945), Ia (102 Bde), Ic (14 Bde) mit Unterlagen über Volkssturm, Zusammenarbeit mit der NSDAP und Bekämpfung von Zersetzungserscheinungen; vom AOK 1 (Saargebiet, Pfalz), AOK 7 (Eifel, Hunsrück), Panzer-AOK 5 (Raum Aachen. Düren), Fallschirm-AOK (Niederlande) gibt es jeweils nur wenige Bände.

Lit.: GUIDES to German Records. vol. 14, 42. 44, 48, 49.

\subsubsection{Chefs der Zivilverwaltung im Operationsgebiet}

Bei Kriegsbeginn wurde die vollziehende Gewalt im Operationsgebiet im Westen wie im Osten dem Oberbefehlshaber des Heeres übertragen. Er übte sie durch Chefs der Zivilverwaltung $(\mathrm{CdZ})$ aus, die den Oberbefehlshabern der Heeresgruppen und Armeen unterstanden. Sie konnten den Zivilbehörden in militärisch relevanten Angelegenheiten - Polizeischutz, Verkehrsregelung, Sach- und Dienstleistungen für die Truppe, Gesundheitsund Veterinärpolizei nach einem ErlaB des Oberbefehlshabers der Heeresgruppe B von November 1939 - Weisungen erteilen und von ihnen Berichte anfordern.

Aus dem Norden des Operationsgebietes sind Akten des dort zum CdZ bestellten Oberpräsidenten von Westfalen und Gauleiters Alfred Meyer aus der Registratur des Oberpräsidiums (MS, 84 Bde, mit Vorakten ab 1936) überliefert. Sie betreffen u. a. Organisation und Geschäftsbetrieb (20 Bde), Mobilmachungsvorbereitungen (11 Bde), Verbindung zu den Kommandobehörden (7 Bde, sämtlich aus der Verwaltungsabteilung). Akten der Polizeiabteilung (12 Bde) enthalten u. a. einen Teil des KTB (AugustOktober 1939) und Befehle des Höheren SS und Polizeiführers West. Von der Wirtschaftsabteilung liegen Akten über Organisation (4 Bde), gewerbliche Wirtschaft (14 Bde), Bewirtschaftungsmaßnahmen und Preisüberwachung (4 Bde) sowie Versorgung der Bevölkerung (5 Bde) vor. 
Ebenfalls aus der Zuständigkeit Meyers als CdZ stammen Anlagen zum KTB der 4. Armee (BA-MA, Best. RH 20-4, 8 Bde). Sie enthalten neben allgemeinen Richtlinien und Plänen für die Evakuierung der Zivilbevölkerung aus dem voraussichtlichen Kampfgebiet im gesamten Wehrkreis VI Vorgänge über Straßen- und Brückenbau, Verkehrssicherung, Wachdienst von Landesschützeneinheiten, Regelung des Straßenverkehrs, Verbot von Ausflugsreisen und erlaubte wie verbotene Ausübung der Jagd durch Wehrmachtangehörige. Vom CdZ beim AOK 5 sind das Kriegstagebuch (24. August 1939Mai 1940) und an ihn gerichtete Gestapoberichte (August-Oktober 1939) vorhanden (BA-MA, Best. RH 20-5).

Aus dem Süden des Operationsgebietes, in dem der Koblenzer Regierungspräsident Mischke für Oberpräsident Terboven die Funktion des $\mathrm{CdZ}$ ausübte, soweit es zum Wehrkreis XII gehörte, sind nur Berichte (September/Oktober 1939) und die Kriegstagebücher der Abteilungen I und II und der Polizeiabteilung (August 1939-April 1940) in Akten des AOK 1 (BA-MA, Best. RH 20-1) überliefert. Berichte der Staatspolizeistellen Köln und Trier an den $\mathrm{CdZ}$ sind als Kopien für das RSHA (BA, Best. R 58), Korrespondenz mit der Regierung Aachen in deren Akten (D-K) erhalten.

\subsubsection{Luftflottenkommando Reich BA-MA, Best. RL 7}

Aus dem Stab der Luftwaffenbefehlshaber Mitte, Generaloberst Weise und Generaloberst Stumpff, denen die Luftstreitkräfte in der Heimat ab 1944 unter der Bezeichnung Luftflottenkommando Reich unterstanden, sind nur wenige Akten überliefert (25 Bde, 1941-1945), darunter ein Tätigkeits- und Erfahrungsbericht (Oktober-Dezember 1941), Denkschriften u. a. über Luftverteidigung (1941), Zusammenstellungen über Feindeinflüge (1941-1945) und Unterlagen über Organisation, Flaktaktik und Nachrichtenwesen (1944-1945). Ergänzungsüberlieferung über den Luftkrieg über dem Reichsgebiet ist in Sammlungen vorhanden (MSg 2).

Lit: K. O. HOFFMANN: Die territoriale und taktische Gliederung der Luftfotte Reich am 1. 2. 1945. 1955. - K.-H. VOLLKER: Die deutsche Heimatluftverteidigung im Zweiten Welıkrieg. 1966.

\subsubsection{Marinegruppenkommandos BA-MA, Best. RM 35 I, II}

Die Kriegstagebücher und Akten der Marinegruppenkommandos West in Wilhelmshaven (bis August 1940) und Ost in Kiel (ab August 1940 Nord in Wilhelmshaven) enthalten einige Informationen über Kriegsvorbereitungen und -ereignisse an den Küsten von Nord- und Ostsee in den ersten Kriegsmonaten. Unterlagen der fuir die Deutsche Bucht und für die westliche Ostsee speziell zuständigen Kommandobehörden sind nur splitterhaft überliefert (Best. RM 45).

\subsection{Militärische Dienststellen mit regionaler Zuständigkeit und festen Standorten im späteren Gebiet der Bundesrepublik Deutschland}

Die schriftliche Überlieferung von Kommandobehörden aller Wehrmachtteile, die für Einheiten und Dienststellen in bestimmten Reichsteilen zuständig waren, von Heeresverbänden in Standorten der Vorkriegszeit und von Truppen der Luftwaffe im Einsatz gegen feindliche Luftangriffe enthält trotz ihres relativ geringen Umfangs zahlreiche 
Informationen für die allgemeine Geschichte von Gebieten und Orten sowie zur Wirschaftsgeschichte. Sie ist nachstehend berücksichtigt, soweit es sich nicht lediglich um Aktensplitter von Wehrmacht- (Best. RW 17), Standort- (Best. RH 34) und FliegerhorstKommandanturen (Best. RL 21). Lazaretten (Bestände RH 55, RM 30) und kleineren Verbänden des Ersatzheeres (Bestände RH 38 und RH 54) handelt, die lediglich bei den Wehrmachtkommandanturen München (21 Bde, 1943-1945) und Würzburg (30 Bde, 1936-1945) etwas größeren Umfang haben (einzelne Garnisons-, Kasernenund Fliegerhorstgeschichten in Best. MSg 2).

\subsubsection{Heeres- und Wehrmachtdienststellen}

\subsubsection{Stellvertretende Generalkommandos und Befehlshaber in den Wehrkreisen BA-MA, Best. RH 53}

Seit 1935 bzw. 1937 war das Reichsgebiet in 13 Wehrkreise eingeteilt, in denen jeweils ein Armeekorps stand, dessen Kommandierender General gleichzeitig als Befehlshaber im Wehrkreis die Spitze der Militärverwaltung mit den für Ersatz und Versorgung zuständigen Dienststellen bildete. Bei Kriegsbeginn traten die aktiven Truppenteile zum Feldheer, die Funktionen im Wehrkreis übernahm ein Stellvertretendes Generalkommando. Vom Schriftgut aus den acht Wehrkreisen für Berlin und die Provinzen und Länder in Nordwest-, West-, Südwest- und Süddeutschland sind folgende Bestände (mit Vorakten der Wehrkreise der Reichswehr) überliefert (vgl. auch Abschnitt 2.1.1.3 zu den Reichsverteidigungskommissaren in den Wehrkreisen), die in allen Fällen Stammtafeln und andere Unterlagen über die unterstellten Einheiten und Dienststellen. vielfach auch über Ausbildung und Mobilmachungsvorbereitungen enthalten; angegeben sind jeweils die Stabsabteilungen ( $\mathrm{vgl}$. oben zu 8.3.1), aus denen sie überwiegend stammen:

Wehrkreis III, Berlin

Vorgänge u. a. über den NS-Führungsoffizier, Entlassung von Juden, Vorbereitung der Verteidigung Berlins (insgesamt 50 Bde, 1933-1945).

\section{Wehrkreis V, Stuttgart}

Ia (18 Bde, 1932-1943), Ib (6 Bde, 1935-1939), Ic (4 Bde, 1937-1944), NS-Führungsoffizier (3 Bde, 1944-1945), IIa (7 Bde, 1927-1943), IV (4 Bde, 1936-1939), KTB des Abschnittskommandos Baden-Baden (Juni 1940), Sektionsprotokolle der Wehrkreisprosektur am Pathologischen Institut der Universität Tübingen (17 Bde, 1941-1945); Akten betr. u. a. Verhältnis zur NSDAP, Luftschutz, Wehrkreisbücherei (insgesamt 68 Bde, 1919-1945), Ergänzungsüberlieferung, u. a. über Kommandeurbesprechungen, im Nachlaß des Befehlshabers (1933-1935) General Curt Liebmann (BA-MA).

\section{Wehrkreis VI, Münster}

KTB mit Anlagen (März-April 1945), Ia (13 Bde, 1935-1945), Ib (12 Bde, 1937-1945), Ic (13 Bde, 1937-1944, dabei 7 Bde des Gräberoffiziers betr. Sterbefälle sowjetischer Kriegsgefangener 1944), Ila (5 Bde, 1924-1945), Militärgeographischer Dienst (4 Bde), Kommandeur des Streifendienstes (4 Bde, 1943-1944), Verwaltungs- und Bausachen (25 Bde), Ausbildung der Landwehr (49 Bde, auch von der Heeresdienststelle 9, Köln), Ergänzungsüberlieferung aus seiner Tätigkeit als Stabschef (1936 im Nachlaß Halder). 


\section{Wehrkreis VII, München}

Der umfangreiche Bestand (insgesamt 1450 Bde, 1919-1945) enthält:

- KTB (Besetzung Österreichs und der Tschechoslowakei und August 1939-Juni 1944),

- Akten aus dem Büro des Kommandierenden Generals Generaloberst Ritter von Schobet (15 Bde, dabei Aufzeichnungen über Besprechungen, Glückwünsche, Verwaltung eines Hilfsfonds), des Chefs des Stabes (6 Bde, mit Anordnungen), des Ia (ca. 150 Bde, dabei 9 Bde betr. innere Sicherheit 1936-1944, ca. 20 Bde betr. Luftschutz 1942-1945), Ib (ca. 50 Bde, vor allem zum Wehrgesetz), der Adjutantur (ca. 25 Bde), des Ila (ca. 600 Bde), Ibb (10 Bde), des Beauftragten für die personelle Ergänzung (20 Bde) und der Verwaltung (ca. 80 Bde), außerdem des Feldzeugkommandos VII, der Heeresdienststelle 10 u. a. nachgeordneter Dienststellen.

Sie enthalten auch Vorgänge aus Ehrengerichts- und Disziplinarverfahren, über Arierbestimmungen, Verhältnis zur NSDAP, Grenzsicherung, die innenpolitische Lage in Österreich ab 1934, Traditionspflege 1939, Luftangriffe, Kriegsgefangene und Kraftfahrwesen. Ergänzungsüberlieferung bieten der NachlaB des Kommandierenden Generals Karl Kriebel (N 670) und Aufzeichnungen seines Vorgängers (1933-1935) General Wilhelm Adam (IfZ, ED 109). Bilder „aus dem Archiv des stellvertretenden Generalkommandos des VII. Korps" wurden 1941 vom Amt Lichtbild der Reichspropagandaleitung der NSDAP (,Die feldgraue Heimat", IfZ) veröffentlicht.

Lit.: K. J. MŪLLER: Reichswehr und „Röhm-Affäre“. 1968. - A. HOCH, H. WEISS: Die Erinnerungen des Generalobersten Wilhelm Adam. 1980.

\section{Wehrkreis IX, Kassel}

Unterlagen u. a. für „Einsatz West“ 1936, über Fürsorge und Versorgungsangelegenheiten, Ausbildung (insgesamt 130 Bde, 1935-1945).

\section{Wehrkreis X, Hamburg}

Ia (23 Bde, 1937-1945), Ila (8 Bde, 1936-1945), Verwaltung, insbesondere der Liegenschaften (68 Bde, u. a. Beschlagnahmen nach dem Reichsleistungsgesetz), dabei einzelne Tätigkeitsberichte und Unterlagen über Personal-, Organisations- und Fürsorgeangelegenheiten, Ergänzungsüberlieferung durch Tagebuchaufzeichnungen 1941-1944 des Stabschefs Oberst Ernst Ebeling bei der Forschungsstelle für die Geschichte des Nationalsozialismus in Hamburg.

\section{Wehrkreis XI, Hannover}

Ia (8 Bde, 1936-1945), Ib (5 Bde), IIa (3 Bde), NS-Führungsoffizier (3 Bde, 1944-1945), Verwaltung (30 Bde), dabei u. a. Unterlagen über Zusammenarbeit mit der NSDAP, wehrgeistige Betreuung, Luftschutz und Verfügungen des evangelischen Wehrkreispfarrers.

\section{Wehrkreis XII, Wiesbaden}

Ia (39 Bde), Ib (10 Bde) Ic (3 Bde), Ila und IVb (je 3 Bde), Verwaltung (35 Bde). Die Akten betreffen u. a. Festungsbau, Sicherung des Rheins, Luftschutz, wehrgeistige Betreuung, Kriegsgefangenenwesen, Fürsorge und Versorgung und enthalten auch das KTB der Quartiermeistergruppe z.b.V. (September-Dezember 1944) und Vorgänge des Generalkommandos der Grenztruppen Saarpfalz (3 Bde, 1939). 


\section{Wehrkreis XII, Nürnberg}

Ia (40 Bde mit KTB über den Einmarsch in Österreich 1938), Ib (ca. 70 Bde), Verwaltung (ca. 40 Bde), ferner 2. T. umfangreiche Akten über Besetzung des Sudetenlandes und der Tschechoslowakei, Grenzbefestigurgen und Grenzwacht (ca. 200 Bde der Heeresdienststelle 6, Regensburg, und ca. 300 Bde der untersteliten Ausbildungsleiter in Deggendorf, Marktredwitz, Regensburg und Weiden bis 1938), femer über Spionageabwehr, Einsatz beim Reichsparteitag, Ausstellung „Unser Heer“, Ausrüstung und Verpflegung (insgesamt 670 Bde, 1931-1945).

\section{Wehrwirtschaftsoffiziere bei den Wehrkreiskommandos}

Von ihnen sind Kriegstagebücher und Tätigkeitsberichte (in Best. RW 46, insgesamt 51 Bde, 1943-1945) überliefert.

Lit: GUDES to German Records. vol. 34 und 38. - G. HOCH. R. SCHWARZ: Verschleppt zur Sklavenarbeit. Kriegsgefangene und Zwangsarbeiter in Schleswig-Holstein [Wehrkreis X]. 1984.

\subsubsection{Rüstungsinspektionen und -kommandos}

BA-MA, Bestände RW 20 und 21

Von den 1935 als Wehrwirtschaftsinspektionen in den Wehrkreisen errichteten Rüstungsinspektionen sind in der Regel die Kriegstagebücher und monatliche Lageberichte sowie jeweils deren Zusammenfassung zu einer „Geschichte der Rüstungsinspektion“ (1939. 1944) überliefert, von den ihnen nachgeordneten Wehrwirtschaftsstellen bzw. Rüstungskommandos die Kriegstagebücher (1939-1944). Sie bieten eine Menge von Informationen über die Wirtschafts- und Versorgungslage und auch über den Arbeitseinsatz von Kriegsgefangenen, Zwangsarbeitern und der Zivilbevölkerung. Sofern die Bestände auch anderes Schriftgut unterschiedlichen Umfangs enthalten, wird in den nachfolgenden Angaben zu den Dienststellen in Berlin und im späteren Gebiet der Bundesrepublik darauf hingewiesen.

Rüstungsinspektion IU (Berlin, 18 Bde) mit Rüstungskommandos Berlin I (9 Bde, 1942-1944), II (11 Bde), III (19 Bde), IV (5 Bde) und V (7 Bde, 1943-1944).

Rüstungsinspektion V (Stuttgart, ab 1942 nur für Württemberg, 20 Bde, KTB Juli 1943 März 1944, Geschichte bis 1941, Monatsberichte 1939-Februar 1942) mit Rüstungskommandos Stuttgart I, II und Ulm (je 3 Bde, 1942-1944).

Rüstungsinspektion Oberrhein (StraBburg, ab 1942 für Baden und ElsaB, 51 Bde, mit Rundschreiben und Akten u. a. über Rheinschiffahn, Lohnpolitik, Arbeitsmarkt, Betriebsverlagerungen, Listen von Industriebetrieben) mit Rüstungskommandos Freiburg (bis 1942 Villingen, 12 Bde) und Mannheim ( 9 Bde).

Rüstungsinspektion VI (Münster, 24 Bde, dabei Verzeichnis von Tanklagern und -stellen) mit Rüstungskommandos Bielefeld (21 Bde, dabei Unterlagen über MobPlanspiel, Kohle- und Treibstoffversorgung, Betriebsverlagerungen), Dortmund (17 Bde), Düsseldorf (43 Bde, dabei Einzelfallakten über Fach- und Schlüsselkräfte, Verlagerung von Aufträgen ins Ausland), Essen (16 Bde, dabei Angaben über Erhebungen in einzelnen Betrieben, Produktionsverluste, Werkschutz), Lüdenscheid (16 Bde), Köln (16 Bde) und Recklinghausen (bis 1943 Osnabrïk, 20 Bde, mit Rundschreiben).

Rüstungsinspektion VII (München, 23 Bde) mit Rüstungskommandos Augsburg (s. u.) und München (7 Bde, 1943-1944).

Rüstungsinspektion IX (Kassel, 27 Bde) mit Rüstungskommando Kassel (17 Bde). Rüstungsinspektion X (Hamburg, 28 Bde) mit Rüstungskommandos Bremen (21 Bde), Hamburg I (11 Bde) und II (6 Bde), Kiel (20 Bde). 
Rüstungsinspektion XI (ab 1943 XIa, Hannover, 34 Bde) mit Rüstungskommandos Braunschweig (13 Bde) und Hannover (9 Bde, 1942-1944).

Rüstungsinspektion XII (ab 1942 XIla, Wiesbaden, 43 Bde, dabei Berichte über Betriebsprüfungen, Werkschutzpläne) mit Rüstungskommandos Frankfurt und GieBen (je 20 Bde), Wiesbaden (3 Bde, 1942-1944).

Rüstungsinspektion XIIb (ab 1942, Saarbrücken, später Metz, 4 Bde) mit Rüstungskommandos Koblenz (bis 1942 zu XII, 9 Bde, 1939-1944), Ludwigshafen (16 Bde, 1942-1945, mit Betriebslisten und Angaben über Lage im Januar 1945, Unterlagen über Kohleversorgung) und Saarbrücken (bis 1942 zu XII, 12 Bde).

Rüstungsinspektion XIII (Nürnberg, 15 Bde) mit Rüstungskommandos Coburg (15 Bde, dabei Akten betr. Verlagerung von Rüstungsbetrieben), Nürnberg ( 9 Bde), Regensburg (3 Bde, 1942-1944) und Würzburg (24 Bde, mit Akten über Arbeitseinsatz und Uk-Stellungen).

\section{Rüstungskommando Augsburg}

Die fast vollständig erhaltene Registratur (ca. 1400 Bde, 1937-1945) umfaBt neben dem Kriegstagebuch (mit einer Lücke für die zweite Hälfte 1942) Geschäftsverteilungs- und Arbeitspläne, Dienstanweisungen, Verfügungen und Rundschreiben, Besprechungs- und Bereisungsprotokolle, Erfahrungs- und Tätigkeitsberichte, Firmenverzeichnisse und Akten über Dienststellenverwaltung, Rechtsangelegenheiten (mit Einzelfällen von Strafverfahren wegen Kriegswirtschaftsverbrechen), Wehrmacht- und private Rüstungsbetriebe (mit Korrespondenzen), Luftangriffe und Kriegsschäden, Spionage- und Sabotageabwehr, Arbeitseinsatz (Einberufungsaktionen und Uk-Stellungen, Kräftebedarfsmeldungen, Kriegsgefangene und Zwangsarbeiter, Rüstungsurlauber der Wehrmacht, Zusammenarbeit mit DAF), Erteilung und Verlagerung von Aufträgen, Fertigungsberichte und -programme (u. a. Jägerprogramm, speziell Messerschmitt-Werke), Rohstoff- und Energieversorgung, Bauwirtschaft, Preisprüfung, Transport und Verkehr, die Zuständigkeit erstreckte sich auf den Regierungsbezirk Schwaben (ohne Kreis Nördlingen) und drei (ab 1942 noch einen) oberbayerische Kreise.

Lit: GUIDES to German Records. vol. 7 und 17. - H. GRIESER: Materialien zur Rüstungswirtschaft Schleswig-Holsteins im Dritten Reich. 1987. - D. KRAUSE-VILMAR: Zur Typik des Terrors gegen ausländische Zwangsarteiter im Bereich des Rüstungskommandos Kassel (1940-1945). 1985. - J. FEAR: Die Rüstungsindustrie im Gau Schwaben 1939-1945. 1987.

\subsubsection{Wehrersatzinspektionen, Wehrbezirkskommandos und Wehrmeldeämter BA-MA, Bestände RW 14 und 15}

Die geringen Reste von Akten der für die Erfassung der Freiwilligen und Wehrpflichtigen und ihre Einberufung zuständigen militärischen Dienststellen (Ergänzungsüberlieferung in Schriftgut der allgemeinen inneren Verwaltung, Abschnitt 2.1) dokumentieren u. a. Mobilmachungsvorbereitungen, Aufstellung der Grenzwacht und anderer Sicherungsverbände, Einberufungen im September 1938, vormilitärische Ausbildung in Zusammenarbeit mit der SA, Entlassungen und Uk-Stellungen. Sie stammen von den Wehrersatzinspektionen Berlin (20 Bde), Kassel (10 Bde), Nürnberg (33 Bde), Regensburg (28 Bde) und fünf weiteren (mit zusammen 11 Bden) und von 86 Wehrbezirkskommandos, z. T. auch ihnen unterstellten Wehrmeldeämtern (460 Bde, 1934-1945), darunter Amberg (11 Bde), Hameln (9 Bde), Kassel II und III (18 Bde), Kempten (A, 10 Bde), Köln (29 Bde), Korbach (7 Bde), Memmingen (A), Mönchengladbach (24 Bde), Nürnberg II (12 Bde), Passau (33 Bde), Regensburg (30 Bde), Straubing (21 Bde), Tauberbischofsheim 
(11 Bde) und Weiden (43 Bde), ferner Wehrmeldeamt Neuwied (13 Bde). Von den bei den Wehrbezirkskommandos geführten Wehrstammbüchern der einzelnen Wehrpflichtigen befinden sich 2,8 Millionen, überwiegend aus dem Rheinland und Westfalen, bei der Zentralnachweisstelle des Bundesarchivs, eine weitere Million fast ausschlieBlich aus Bayern bei der Deutschen Dienststelle (WASt) Berlin.

Lit.: R. ASOLON: Wehrgeselz und Wehrdienst 1935-1945. 1960.

\subsubsection{Wehrmachtfürsorge- und Versorgungsdienststellen}

BA-MA, Best. RW 16

Überliefert sind lediglich Aktenreste (auch Einzelfälle, insgesamt 350 Bde, 1928-1945) der Wehrmachtversorgungsgruppe VII (München), des Wehrmacht-Fürsorge- und Versorgungsamtes München-Landshut, der Fürsorgeoffiziere Lüneburg und Stade u. a. über die Umschulung von Verwundeten, Unterbringung von Versorgungsanwärtern im öffentlichen Dienst, andere Berufsförderungs- und FürsorgemaBnahmen bei Entlassung von dienstunfähigen Berufssoldaten sowie einzelne Vorgänge von acht weiteren Dienststellen im Gebiet der späteren Bundesrepublik Deutschland.

\subsubsection{Kriegsgefangenenlager BA-MA, Best. RH 49}

Von den Kriegsgefangenenlagern im Reichsgebiet, die den Stellvertretenden Generalkommandos in den Wehrkreisen unterstanden (Stammtafeln in der Regel in deren Akten), ist wenig überliefert. Aus dem späteren Gebiet der Bundesrepublik Deutschland stammen Aktenreste der Offizierslager (Oflag) VII D, Tittmoning, und XI A, Osterode/Harz, und der Mannschafts-Stammlager (Stalag) VI A, Hemer, VI G, Köln, VII A, Mosburg, XII A, Limburg/Lahn, XII D, Trier, XII F, Forbach und XIII A, Sulzbach/Oberpfalz, ferner das Kriegstagebuch des Abwehroffiziers im LuftwaffenDurchgangslager (Dulag) Oberursel (Best. RL 24. Mai 1942-Mai 1943, Ergänzung dazu in MSg 2). Verbrechen an Kriegsgefangenen im Stalag XIII C, Bayreuth, waren Gegenstand eines Verfahrens vor einem englischen Militärgericht (BA, Best. AllProz 8).

Lit: Y. DURAND: La vie quotidienne des prisonniers de guerre dans les Stalags. les Oflags et les Kommandos 1939-1945. 1987. - G. MOKRY: Zur Funktion und Organisation der faschistischen Kriegsgefangenenlager im II. Weltkrieg. 1972.

\subsubsection{Abwehrstellen}

BA-MA, Best. RW 49

Während von den Abwehrstellen in den Wehrkreisen III, V, VI und IX nur einzelne Aktensplitter überliefert sind, von den Abwehrstellen in den Wehrkreisen XI, XII und XIII gar nichts, blieben von der Abwehrstelle VII, München, vor allem Akten über Beschaffung von Nachrichten aus der Tschechoslowakei erhalten (40 Bde, 1934-1938), und aus dem Wehrkreis $\mathrm{X}$ liegen Akten der Abwehrnebenstelle Bremen vor (Mikrofilme von 200 Bden, 1935-1943); sie enthalten u. a. Personalakten und -karteien, Berichte und Meldungen von Agenten, darunter Angehörige der Besatzung deutscher und ausländischer Handelsschiffe, aus den USA, Argentinien, Brasilien, Mexiko, den übrigen amerikanischen und einzelnen europäischen Küstenstaaten.

Lit: L. FARAGO: Das Spiel der Füchse. 1972. - U. BRAMMER: Spionageabwehr und .Geheimer Meldedienst". 1989. 


\subsubsection{Dienststellen und Stäbe der Landesbefestigung}

BA-MA, Best. RH 32

Der Bestand vereinigt wenige Akten des Inspekteurs der Landesbefestigungen West (12 Bde, 1943-1945) mit Schriftgut des Höheren Kommandos Saarpfalz (11 Bde), der Kommandanturen der Befestigungen Eifel, Niederrhein (KTB 1939-1940), Oberrhein (1940) und Saarpfalz und der Festungsdienststellen Trier, Düren, Karlsruhe, Helgoland, Traben-Trarbach und Pirmasens. Den Hauptanteil haben Akten und Bauunterlagen des Festungspionierstabs 10, Heilbronn, über den Bau der Neckar-Enz-Stellung (ca. 2000 Bde, ab 1934) und des Festungspionierstabs 14, Aschaffenburg, über den Bau der Wetterau-Stellung (ca. 400 Bde), ferner Unterlagen von Wehrgeologengruppen.

Lit.: D. BETTINGER, M. BÜREN: Der Westwall. 2 Bde. 1990.

\subsubsection{Divisionskommandos vor 1939}

BA-MA, Best. RH 26

Von den 24 Divisionen des Friedensheeres mit Standorten im späteren Gebiet der Bundesrepublik Deutschland ist relativ wenig überliefert. Neben Aktensplittern der 20. (Hamburg, 4 Bde), 22. (Bremen, 14 Bde, 1936-1939), 30. (Lübeck), 33. (Darmstadt), 34. (Koblenz) und 35. (Karlsruhe) Infanteriedivision und der 1. Gebirgsdivision (GarmischPartenkirchen, Unterlagen über Einmarsch in Österreich 1938) sind folgende Bestände vorhanden:

\section{Infanteriedivision, München}

Die Akten (bis Oktober 1934 zugleich Wehrkreiskommando VII) des Kommandeurs (mit Korrespondenz von Generalmajor Halder 1934-1936) und der Adjutantur (46 Bde, 1933-1939) enthalten Besprechungsniederschriften und betreffen u. a. das Verhältnis zur SA (dabei „Röhm-Putsch“ 1934), zur NSDAP, SS und HJ, Bekämpfung von Spionage und kommunistischer Zersetzung, Sicherung der Grenze zu Österreich 1934-1938. Der übrige Bestand stammt aus folgenden Sachgebieten:

Organisation und Heeresaufbau (ca. $80 \mathrm{Bde}$ ); Mobilmachungsplanung (108 Bde); Militärgeographie (24 Bde); Haushalt (15 Bde); allgemeine (49 Bde) und Offizierspersonalsachen (ca. 50 Bde); Ausbildung und Manöver (ca. 150 Bde, davon 2 bzw. 4 Bde Kriegstagebücher und 10 bzw. 34 Bde Akten über den Einmarsch in Österreich und im Sudetenland 1938); Sanitäts- (11 Bde) und Veterinärwesen (14 Bde); Ausrüstung mit Bekleidung, Verpflegung, Waffen, Munition und Gerät (ca. 100 Bde).

\section{Infanteriedivision, Regensburg}

$\mathrm{Zu}$ den allgemeinen Akten (30 Bde) gehören Tagesbefehle und Vorgänge über SA, Arbeitsdienst, Ehrenangelegenheiten, Traditionspflege, die Lage in Österreich (auch 5 Bde Kriegstagebuch 1938), Maßnahmen gegen Juden, Kommunisten und Bibelforscher. Den Hauptteil des Bestandes bildet Schriftgut über Organisation (ca. 80 Bde), Mobilmachung (32 Bde), Ausbildung und Manöver (ca. 80 Bde), Personalangelegenheiten im allgemeinen (55 Bde) und der Offiziere und Beamten (ca. 70 Bde).

\section{Infanteriedivision, Nürnberg}

Vorhanden sind Stabs- und Tagesbefehle (30 Bde, dabei Rundschreiben der DAFAbteilung Wehrmacht 1937-1939), Korrespondenz der Kommandeure Ritter v. Schobert, Haase, Friderici und ihrer Adjutanten (12 Bde), Vorgänge über Innen- und AuBenpolitik (27 Bde, dabei über SA, HJ, Ehrenangelegenheiten, Traditionspflege), den Einmarsch 
in Österreich (21 Bde) und im Sudetenland (2 Bde) und Akten über Organisation und inneren Dienst (ca. 200 Bde, dabei 12 Bde betr. Reichsparteitage 1934-1939), Haushalt (ca. 40 Bde), Ausbildung und Übungen (ca. 100 Bde), Sport (12 Bde), allgemeine Personal- (ca. 100 Bde) und Disziplinarsachen (37 Bde, mit Tatberichten), Ausstattung mit Gerät, Kraftfahrzeuge, Waffen und Bekleidung (ca. 90 Bde).

\subsubsection{Truppenteile vor 1939 BA-MA, Best. RH 37, 46 und 50}

AusschlieBlich aus Bayern (Wehrkreis VII bzw. XIII) stammen Akten folgender Einheiten (mit Vorakten der Reichswehr):

Infanterieregimenter Nr. 19 (München, ca. 200 Bde, 1920-1939, u. a. betr. Verhältnis zu NSDAP-Funktionären, Besetzung Österreichs, Luftschutz, Abwehr), Nr. 20 (Regensburg, 30 Bde, 1920-1938, dabei Vorgänge über SA-Lager, Österreich), Nr. 61 (München und Traunstein, ca. 120 Bde, 1936-1942, u. a. betr. innere Sicherheit, Abwehrangelegenheiten, Zusammenarbeit mit NSKK und HJ) und Nr. 63 (Ingolstadt und Neuburg/Donau, ca. 150 Bde, 1935-1940, u. a. Geheimakten, Unterlagen über vormilitärische Ausbildung und „Tag der Wehrmacht"),

Pionierbataillone Nr. 7 (München, ab 1936 Rosenheim, 30 Bde, 1927-1939, mit Geheimakten) und Nr. 47 (München, ca. 70 Bde, 1937-1940, u. a. betr. Österreich 1938, Abwehrangelegenheiten, Übungen),

Sanitätsabteilung der 7. Infanteriedivision (München, 30 Bde, 1935-1939).

\subsubsection{Luftwaffe}

\subsubsection{Luftgaukommandos}

BA-MA, Best. RI 19

Den Wehrkreisen des Heeres entsprachen seit 1936 - anfangs mit derselben territorialen Zuständigkeit - Luftgaue, deren Befehlshabern neben der Bodenorganisation der Luftwaffe die leichten Jagdfliegerverbände, die gesamte Flak, Luftschutz, Flugmeldedienst und Nachschub unterstanden, wobei jedoch z. T. mehrere Wehrkreise einen Luftgau bildeten. Überliefert sind folgende Bestände:

\section{Luftgaukommando III, Berlin}

Aktensplitter über Luftangriffe 1940, Organisation und Verwaltung (5 Bde, 1941-1944).

\section{Luftgaukommando V, Stuttgart}

Reste von Verwaltungsakten (4 Bde, 1937, 1944), Unterlagen über Luftangriffe 1944.

\section{Luftgaukommando VI, Münster}

Die Akten (35 Bde, 1937-1943) betreffen Nachrichtenverbindungen, Luftschutz (mit Berichten über Betriebe der Fa. Krupp in Essen), Sanitätswesen und Personalangelegenheiten, weitere über Grundstücke für Zwecke der Luftwaffe, insbesondere Fliegerhorst Lippstadt, sind als Vorakten des Bundesvermögensamtes Soest überliefert (MS, ca. 65 Bde, 1936-1943).

\section{Luftgaukommando VII, München}

Der Bestand (insgesamt ca. 240 Bde) enthält das KTB mit Anlagen (33 Bde, Besetzung des Sudetenlandes 1938, August 1939-Dezember 1944) und Akten u. a. über wehrgeistige Führung (6 Bde, 1940-194), Luftschutz (12 Bde), Abwehr von Zersetzungserscheinungen, Zusammenarbeit mit der NSDAP (6 Bde, 1940-1943), Wetterdienst (33 
Bde), Einsatz, auch der Luftwaffenhelfer (7 Bde) und der SS-Nebelabteilung Obersalzberg, Behandlung italienischer Militärinternierter und Soldaten ( 9 Bde, 1944-1945), Ausbildungs- (14 Bde), Quartiermeister- (43 Bde), Verwaltungs- (26 Bde) und Personalangelegenheiten (11 Bde), über diese auch Korrespondenz des Kommandeurs, General der Flakartillerie Zenetti (5 Bde, 1942-1944).

Luftgaukommando XI, Hannover, ab 1940 Hamburg

KTB der Führungsgruppe (August-Dezember 1939) und des Stabes (April-August 1942), Geschichte des Luftgaues (Aufzeichnung des Befehlshabers General der Flieger Wolff, 3 Bde, 1945), Akten der Abteilungen Ia (11 Bde) und Ic mit Erfahrungsbericht über die Angriffe auf Hamburg im August 1943 und Unterlagen über Luftverteidigung sowie über Werbung für die russische Befreiungsarmee 1944, des Quartiermeisters (15 Bde) und der Verwaltung (8 Bde).

\section{Luftgaukommando XII, Wiesbaden}

Luftlagemeldungen (10 Bde, Juli 1940-Juni 1943), Ic-Lageberichte (4 Bde, 1941-1942), Akten betr. Luftschutz (4 Bde, 1940-1942, u. a. Frankfurt, Kassel, Nürnberg), Organisation und Dienststellenverwaltung (19 Bde, davon 17 Bde Tagesbefehle, 1939-1944).

\section{Luftgaukommando XIV, Nürnberg}

KTB (7 Bde, September 1944-April 1945), Tages- und andere Befehle, vor allem in Organisations- und Personalangelegenheiten (8 Bde, 1944-1945).

Lit.: GUIDES to German Records. vol. 24.

\subsubsection{Kommandobehörden und Verbände der Jagdflieger im Reichsgebiet BA-MA, Bestände RL 8 und 10}

Die Überlieferung beschränkt sich auf Aktenreste des Jagdfliegerführers Süddeutschland (12 Bde betr. Tag- und Nachtjagd im Raum München-Nürnberg-Stuttgart 1943), der 2. (Provinz Hannover, 4 Bde, 1943-1945) und 3. Jagddivision (deutsch-niederländisches Grenzgebiet, 7 Bde mit Teilen des KTB, 1944) und der Jagdgeschwader Nr. 1 (Nordwestdeutschland, 3 Bde, 1942-1944), Nr. 26 (desgl., 4 Bde, 1944-1945), Nr. 53 (Westdeutschland, 2 Bde, 1939-1940) und Nr. 77 (Westdeutschland und Berlin, 19391940), der Nachtjagdgeschwader Nr. 1 (Westdeutschland, 6 Bde, 1944-1945), Nr. 6 (Süddeutschland, 2 Bde, 1944-1945) und Nr. 11 (West- und Nordwestdeutschland, 2 Bde, 1944-1945) sowie des Zerstörergeschwaders Nr. 26 (Süddeutschland, 2 Bde, 1944).

\subsubsection{Flughafenbereichskommandos}

BA-MA, Best. RL 20

Teile von Kriegstagebüchern und einzelne Akten betreffen den Luftkrieg über West-, Nord- und Süddeutschland. Sie stammen von den jeweils für mehrere Flugplätze zuständigen Kommandos in Böblingen, Delmenhorst, Flensburg (mit Vor- und Nachakten von Lübeck-Blankensee und Schleswig), Gelnhausen, Lüneburg (später Stade), Schwäbisch Hall und Werl. 


\subsubsection{Kommandobehörden und Einheiten der Flakartillerie im Reichsgebiet BA-MA, Bestände RL 11 und 12}

Die jeweils nur aus wenigen Bänden mit Teilen von Kriegstagebüchern, Abschußmeidungen, Befehlen, Dienstplänen und Verwaltungsunterlagen bestehende Überlieferung vermittelt insgesamt einen Eindruck von der Bekämpfung der Luftangriffe. Sie stammt vom Flugabwehrkommando Schwarzwald (25 Bde, 1939-1940), der

1. (Berlin, 3 Bde, 1940-1944), 4. (Hanau, 1941-1943), 5. (Südwestdeutschland), 7. (Köln, 1941-1942), 8. (Nordwestdeutschland), 21. (Südwestdeutschland) und 26. Flakdivision (München, 1944), den

Flakregimentern 5 (Krefeld, 1939-1942), 14 (Köln, 1944-1945), 16 (Hamburg, 1944), 24 (Düsseldorf, 1944), 25 (Hannover, KTB 1941-1942), 26 (Bremen, 1941), 29 (Frankfurt, 1943-1944), 46 (Dorsten, 1943-1944), 47 (Münster, 1944-1945), 49 (Mannheim, 1941), 51 (Hamburg, 1944), 54 (Münster, 1943-1944), 60 (Hamburg, 1940), 66 (Augsburg, 1942), 75 (Sturtgart, 1941-1942), 93 (Nürnberg, 1942-1943), 122 (Lübeck. 1942), 139 (Stuttgart, 1945), 155 (Augsburg 1945) und 179 (Schweinfur, 1944-1945) und den

Scheinwerferregimentern Nr. 2 (Schleswig, Augsburg, 1942), 3 (Münster, 1943), 74 (Essen, Duisburg, 1941-1942) und 611 (Ruhrgebiet, 1940-1942) und von

schweren und leichten Flak- sowie Scheinwerferabteilungen in Augsburg (19411942, 1944-1945), Berchtesgaden (1944), Berlin (1944), Bremen-Neuland (1945), Duisburg (1943-1945), Frankfurt (1944-1945), Gelnhausen (1944), Hagen (1943), Hamburg (1939-1945), Hanau (1939-1945), Herne (1943-1944), Homburg/Saar (1944), Kassel (1945), Köln (1944-1945), Krefeld (1942), Lübeck (1942-1943), Marl (1942), München (1942-1944), Nürnberg (1944), Peine (1941), Rheydt (1940), Ruhrgebiet (1940-1942), Schweinfurt, Sylt (1941-1942), Wesseling (1944), Wuppertal (1942).

Die Anfänge der Flaktruppe und die Vorbereitung auf den Luftkrieg sind in Akten des Flakregiments 12, Berlin-Lankwitz, dokumentiert (83 Bde, 1933-1939, dabei auch zum Verhältnis zur NSDAP).

Lit.: H.-A. KOCH: Fak. Die Geschichte der deutschen Fakarillerie 1935-1945. 1954.

\subsubsection{Luftschutzregimenter und -abteilungen BA-MA. Best. RL 13}

Nur wenige Akten des Luftschutzregiments 7 über seinen Einsatz in Bayern (14 Bde, 1944-1945), Übungsunterlagen der Abteilung (mot) 23 (Gelsenkirchen, 1943) und Kriegstagebücher der Abteilungen 34 (u. a. Berlin, Hamburg, 1940-1944), 40 (Berlin, 1944) und 48 (Berlin, Hamburg 1944) sind von dieser Truppe vorhanden, die 1942 aus den 1939 zur örtlichen Bekämpfung der Folgen von Luftangriffen gebildeten Einheiten des SHD (Sicherheits- und Hilfsdienst) hervorgegangen ist.

\subsubsection{Bau- und Ausrüstungsdienststellen und -einheiten}

BA-MA, Bestände RL 23 und 24

Die Bestände enthalten Teile von Kriegstagebüchern des Luftwaffen-Oberbaustabs Schleswig (1939-1940), der Bauregimenter VII (Süddeutschland, 1940) und XII (Kastellaun und Wiesbaden, 1939-1940) und der Luftzeuggruppen 3 (Berlin, 1939-1943), 6 (Münster, 1939-1943), 7 (München, 1943-1944), 11 (Hamburg, 1942-1943), 12 (Wiesbaden 1939-1943) und See (Kiel, 1939-1945). 


\subsubsection{Kriegsmarine}

\subsubsection{Marinestationen der Ostsee und Nordsee}

BA-MA, Bestände RM 31 und 33

Die Kommandierenden Admirale der beiden Marinestationen in Kiel und Wilhelmshaven (ab 1943 Marineoberkommandos Ost und Nord) hatten den Wehrkreisbefehlshabern vergleichbare Aufgaben (für den Ersatz die jeweiligen 2. Admirale). Die Bestände enthalten überwiegend Schriftgut der Kaiserlichen Marine. Aus der Zeit nach 1933 sind lediglich die Kriegstagebücher (Ostsee 16 Bde bis 31. 12. 1944, Nordsee 8 Bde bis 31. 1. 1945, darin Angaben über Luftangriffe auf Kiel und Wilhelmshaven, Heranziehung von Zivilisten zur Küstenverteidigung 1942-1943, Verhältnis zur dänischen Kriegsmarine) und wenige Akten u. a. über Verbandsübungen und sonstige Manöver in der Nordsee (9 Bde, 1928-1939), über Dienststellenverwaltung durch die Marineintendantur bzw. Oberverwaltungsstab Ost (6 Bde) und ein Band Generalakten des Marinechefrichters Ostsee vorhanden.

Einen eigenen Bestand (RM 26) bilden die Akten des Evangelischen Marinestationspfarrers (Marinedekan) Wilhelmshaven (80 Bde, 1921-1945) aus allen Bereichen der Militärseelsorge und ihrer Organisation, ergänzt durch die „Mitteilungen für die evangelischen Pfarrer der Kriegsmarine“ (1940-1945).

Lit.: INVENTAR staatlicher Akten. 1988. Bd. 2 und 3.

\subsubsection{Kriegsmarinedienststellen Hamburg und Bremen}

BA-MA, Best. RM 108

Vorhanden sind Kriegstagebücher und Akten über die Bereitstellung und Bemannung von Hilfsschiffen und die Durchführung von Truppen- und Nachschubtransporten. 
